\title{
Nonlinear Control of an Active Magnetic Bearing System Achieved Using a Fuzzy Control with Radial Basis Function Neural Network
}

\author{
Seng-Chi Chen, ${ }^{1}$ Van-Sum Nguyen, ${ }^{1}$ Dinh-Kha Le, ${ }^{1}$ and Nguyen Thi Hoai Nam ${ }^{2}$ \\ ${ }^{1}$ Department of Electrical Engineering, Da-Yeh University, Changhua 51591, Taiwan \\ ${ }^{2}$ Department of Electrical Engineering, Hue Industrial College, Hue 47000, Vietnam
}

Correspondence should be addressed to Seng-Chi Chen; amtf.csg@msa.hinet.net

Received 14 May 2014; Revised 27 August 2014; Accepted 10 September 2014; Published 12 November 2014

Academic Editor: Xinkai Chen

Copyright (C) 2014 Seng-Chi Chen et al. This is an open access article distributed under the Creative Commons Attribution License, which permits unrestricted use, distribution, and reproduction in any medium, provided the original work is properly cited.

\begin{abstract}
Studies on active magnetic bearing (AMB) systems are increasing in popularity and practical applications. Magnetic bearings cause less noise, friction, and vibration than the conventional mechanical bearings; however, the control of AMB systems requires further investigation. The magnetic force has a highly nonlinear relation to the control current and the air gap. This paper proposes an intelligent control method for positioning an AMB system that uses a neural fuzzy controller (NFC). The mathematical model of an AMB system comprises identification followed by collection of information from this system. A fuzzy logic controller (FLC), the parameters of which are adjusted using a radial basis function neural network (RBFNN), is applied to the unbalanced vibration in an AMB system. The AMB system exhibited a satisfactory control performance, with low overshoot, and produced improved transient and steady-state responses under various operating conditions. The NFC has been verified on a prototype AMB system. The proposed controller can be feasibly applied to AMB systems exposed to various external disturbances; demonstrating the effectiveness of the NFC with self-learning and self-improving capacities is proven.
\end{abstract}

\section{Introduction}

Magnetic bearings are electromechanical devices that use magnetic forces to completely levitate a rotor or suspend it in an air gap without physical contact. Because the system undergoes no friction or wear, it requires no lubrication. In addition, magnetic bearings do not pollute the environment, have a long working life, and can be used in a wide range of applications in aerospace, energy, transportation, and other high technology fields, as well as in high speed ultraprecision machine tools [1-3]. By contrast, conventional mechanical rotary bearings are in physical contact with the shaft, resulting in friction between the shaft and bearings. The temperature of the bearings increases greatly at high rotating speeds, resulting in substantial energy loss and eventual wearing down of the bearings. However, it is difficult to design active controls for magnetic bearing systems because of their high nonlinearity and unstable open-loop electromagnetic dynamics. The suspending structure of a magnetic bearing differs from that of a conventional bearing. Because no contact occurs between the shaft and the bearing, nearly no friction occurs in the AMB system. Moreover, its stiffness and damping can be changed through rotor displacement and velocity feedback control.

In recent years, many intelligent control techniques, such as fuzzy control, adaptive PID control, neural networks control, adaptive fuzzy control, and other control methods, have been developed and applied to the position control for AMB system [4-9]. Although fuzzy control has been successfully applied in several industrial automations, it is not an easy task to obtain an optimal set of fuzzy membership functions and rules in FLC. RBFNNs are used in a variety of applications such as pattern recognition, nonlinear identification, and control time series prediction. In this study, an AMB that supports a ventilator rotor was investigated to ensure the absence of friction and wear between the rotor and stator and improve the life of the ventilator and its rate of rotation. This paper proposes a method for controlling the position 


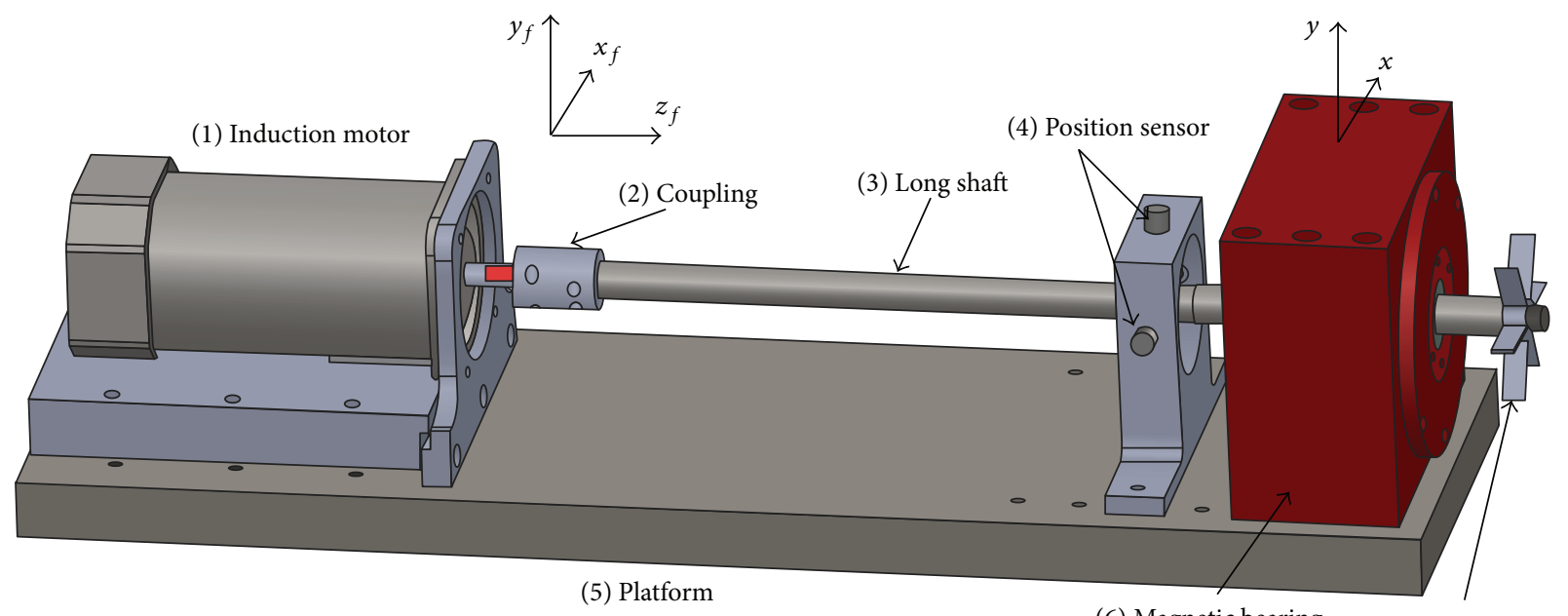

(6) Magnetic bearing

(7) Ventilator

FIGURE 1: Architecture of a ventilator magnetic bearing system.

of the rotor by using the neural fuzzy controller (NFC) approach. The method employs a fuzzy controller system with radial basis function neural network (RBFNN) rotation to identify the AMB system by Jacobian transformation [1014]. The parameters of fuzzy logic controller (FLC) can be optimally tuned to solve the problem of unbalanced vibration in the AMB system by applying the gradient descent method [15] and the real time values according to the AMB system information. The experimental results of the AMB system indicated a satisfactory control performance without overshoot and with no steady-state errors. This control system also satisfies the requirements of real time response and stability of the control AMB system under disturbances.

The remainder of this paper is organized as follows. Section 2 introduces the principle and mathematical model of the AMB system. Section 3 discusses the dynamics of the AMB system. A motion of equation is formulated whereby characteristics of the rotor for rolling and pitching motions and interference between the horizontal and vertical directions can be analyzed. Section 4 presents the structure of a fuzzy controller based on RBFNN identification of the position control loop for mechanical model of the AMB system. Section 5 presents the stability analysis for the AMB system. Finally, Section 6 presents the results and discussion, and Section 7 concludes the paper.

\section{Structure and Mathematical Model of the Active Magnetic Bearing System}

Many recent studies on magnetic bearings have focused on AMBs. The AMB system proposed in this paper is presented in Figure 1. The system included a ventilator, a rotor shaft, a magnetic bearing, a coupling device, and a driving motor. The drive system of the AMB system included differential driving mode power amplifiers and an analog to digital (A/D) converter, as indicated in Figure 2. Two sensors were positioned to measure the displacement of the rotor from the reference position horizontally and vertically. The A/D converter converted the analog signal received from the position sensors into a digital signal. The NFC used this signal as an input, generated a control effort according to measurements, and conducted it using power amplifiers. Four actuating magnets held the rotor at the reference position and transformed the control signals into magnetic forces. As indicated in Figure 2, two pairs of electromagnetic coils were installed perpendicular to the E-shaped stators. These coils produced attractive electromagnetic forces in the perpendicular direction in response to direct currents. All coils installed in the AMBs had the same turns and were symmetrical and uncoupled. For two degrees of freedom, two opposing electromagnets operated in a differential driving mode [16].

As indicated in Figure 2, an electromagnetic force was applied to the rotor along the $x$ - or $y$-axis to keep the rotor in the center of the AMB. The variable $i_{b}$ is the bias current and $i_{x}$ and $i_{y}$ are control currents along the $x$ - and $y$-axes, respectively; $x_{1}$ and $y_{1}$ are the rotor displacements at the magnetic bearing. Following Schweitzer [16-18], the total nonlinear attractive electromagnetic forces along the $x$ - and $y$-axes are given as follows:

$$
\begin{aligned}
& f_{2 x}-f_{1 x}=k\left(\frac{\left(i_{b}+i_{x}\right)^{2}}{\left(x_{g}-x_{1}\right)^{2}}-\frac{\left(i_{b}-i_{x}\right)^{2}}{\left(x_{g}+x_{1}\right)^{2}}\right), \\
& f_{1 y}-f_{2 y}=k\left(\frac{\left(i_{b}+i_{y}\right)^{2}}{\left(y_{g}-y_{1}\right)^{2}}-\frac{\left(i_{b}-i_{y}\right)^{2}}{\left(y_{g}+y_{1}\right)^{2}}\right) .
\end{aligned}
$$

Figure 3 presents a three-dimensional plot of the magnetic force, which is nonlinearly related to the current and the rotor displacement. 


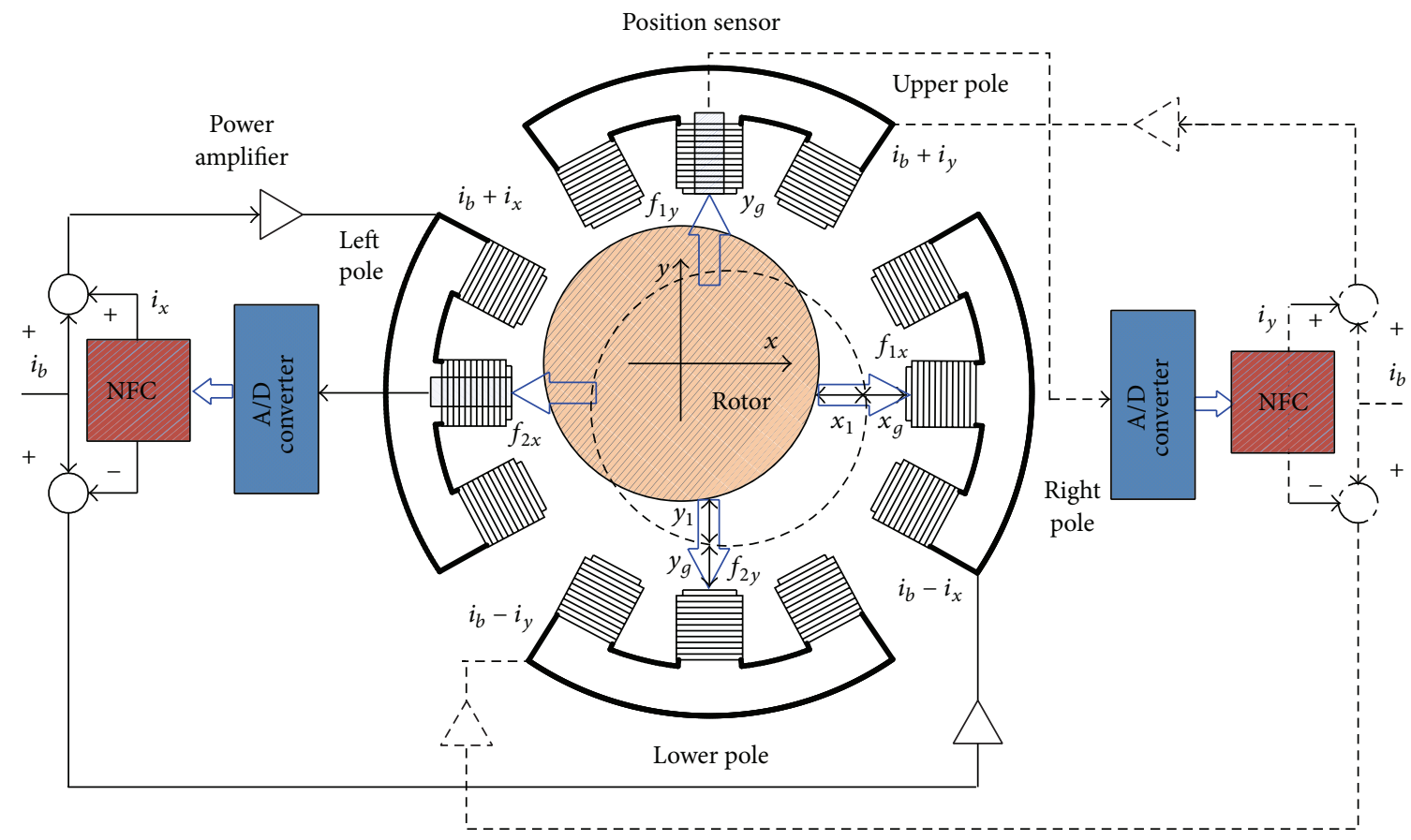

Figure 2: Drive system of an AMB.

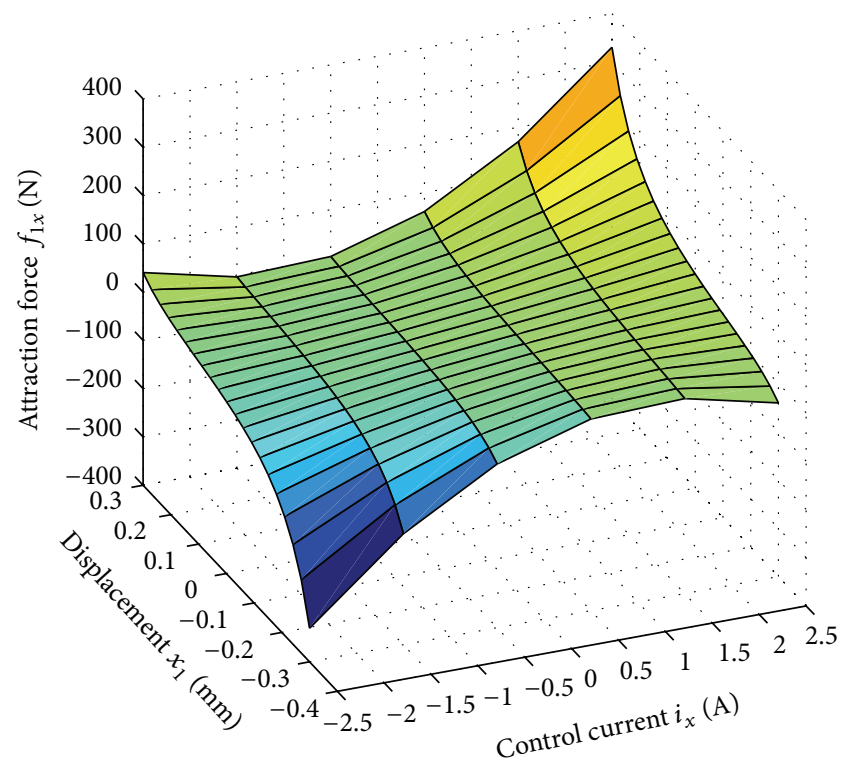

Figure 3: Characteristics of electromagnetic force.

A Taylor expansion is performed and linearization is conducted using (1), yielding the following equations that pertain to the $x$-axis:

$$
\begin{gathered}
k_{i s}=\left.\frac{\partial f_{1 x}\left(x_{1}, i_{x}\right)}{\partial i_{x}}\right|_{x_{1}=0, i_{x}=0}=\frac{4 k i_{b}}{x_{g^{2}}}, \\
k_{d s}=\left.\frac{\partial f_{1 x}\left(x_{1}, i_{x}\right)}{\partial x_{1}}\right|_{x_{1}=0, i_{x}=0}=\frac{4 k i_{b}^{2}}{x_{g^{3}}},
\end{gathered}
$$

where $k_{i s}$ and $k_{d s}$ are the displacement and current stiffness parameters of the magnetic bearings, respectively. In this AMB system, the coil on the $x$ - and $y$-axes circulates the same bias current $\left(i_{b}\right)$. Because the nominal air gaps along the $x$ and $y$-axes are also the same $\left(x_{g}=y_{g}\right)$, the position and current stiffness parameters $k_{i s}$ and $k_{d s}$ that are obtained from the $x$-axis are the same as those obtained for the $y$-axis.

\section{Dynamic Model of Active Magnetic Bearing System}

Rotor dynamics is a crucial aspect of magnetic bearings, particularly at high speeds. It refers to the results of classical vibration theory and gyromechanics; we can know the phenomena of natural vibration, forward and backward whirl, critical speed, precession, and gyroscopic effect, and the dynamic equation for the horizontal shaft magnetic bearing is studied, and the control schemes are also discussed [1921]. In this section, the dynamic equation of the proposed AMB system is derived. An electric AMB, whose rotor and electromagnet are not in contact, was studied. The shaft was suspended horizontally by magnetic forces at one side while being connected to a driving motor with a flexible coupler at the other side. A coordinate of a fixed frame $\left(x_{f} y_{f} z_{f}\right)$ was defined as indicated in Figure 4.

The coordinate $x_{f}$ is in the coupling horizontal direction, $y_{f}$ is perpendicular to the $x_{f}$-axis, and the $z_{f}$-axis coincides with the rotor's axis. Four magnetic attractive forces, $f_{1 x}, f_{2 x}$, $f_{1 y}$, and $f_{2 y}$, are exerted on the rotor along the $x_{f}$ - and $y_{f^{-}}$ axes. The total external forces exerted on the rotor are denoted by $f_{e x}, f_{e y}$, and $f_{e z}$, and the variables $F_{x 1}$ and $F_{x 2}$ are coupling forces. 


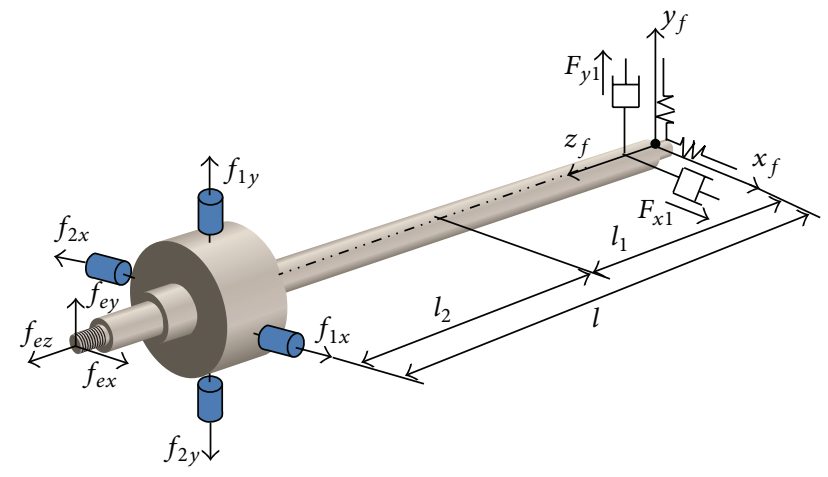

FIgURE 4: Coordinate of a fixed frame.

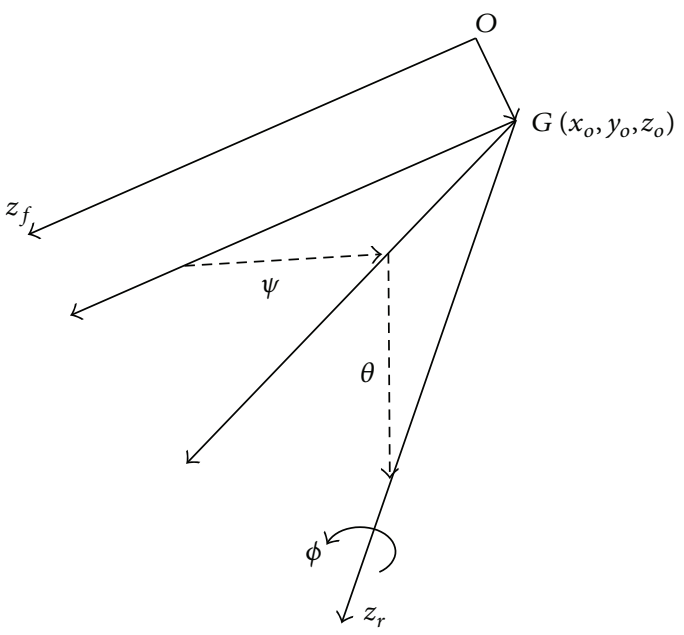

FIGURE 5: Coordinates of a rotor.

3.1. The Transformation between Rotor and Fixed Coordinate of Rotor Dynamic. The fixed frames are related to the rotor coordinates, as indicated in Figure 5 . The $z_{r}$-axis is obtained by rotating the $z_{f}$-axis by $\psi$ on the horizontal plane and by $\theta$ on the vertical plane. The rotating frame with regard to the fixed frame of reference, $x_{o}, y_{o}$, and $z_{o}$, shows the location of $G$ with respect to the fixed frame. When the rotor rotates by $\phi$ around the $z_{r}$-axis, the $x_{r}$ - and $y_{r}$-axes also rotate. Therefore,

$$
\begin{aligned}
& x_{r}=x_{o} \cos \phi+y_{o} \sin \phi, \\
& y_{r}=-x_{o} \sin \phi+y_{o} \cos \phi .
\end{aligned}
$$

For a convenient expression, (3) can be rearranged in the matrix form as follows:

$$
\left[\begin{array}{l}
x_{r} \\
y_{r}
\end{array}\right]=\left[\begin{array}{cc}
\cos \phi & \sin \phi \\
-\sin \phi & \cos \phi
\end{array}\right]\left[\begin{array}{l}
x_{o} \\
y_{o}
\end{array}\right]
$$

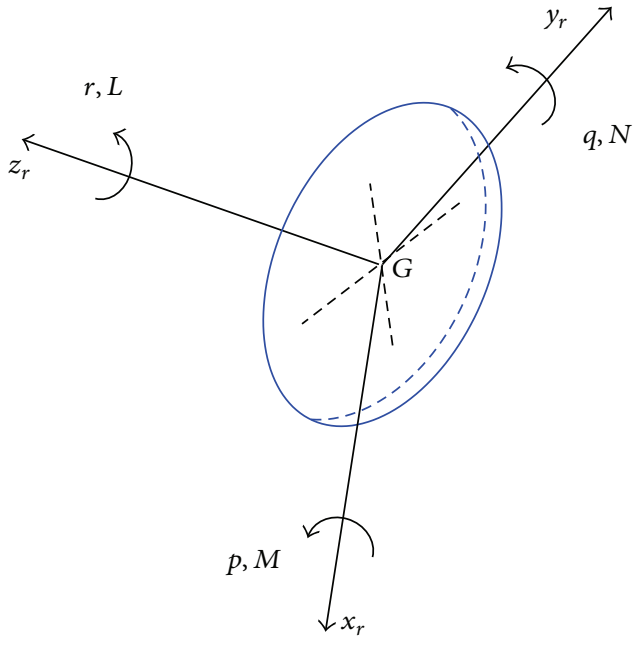

FIGURE 6: Rotor in moving frame of reference.

To achieve an effective control result, $\psi$ and $\theta$ are assumed to be negligible. Hence,

$$
\begin{aligned}
& {\left[\begin{array}{l}
u \\
v \\
w
\end{array}\right]=\left[\begin{array}{ccc}
\cos \phi & \sin \phi & 0 \\
-\sin \phi & \cos \phi & 0 \\
0 & 0 & 1
\end{array}\right]\left[\begin{array}{l}
\dot{x}_{o} \\
\dot{y}_{o} \\
\dot{z}_{o}
\end{array}\right],} \\
& {\left[\begin{array}{l}
p \\
q \\
r
\end{array}\right]=\left[\begin{array}{ccc}
\cos \phi & \sin \phi & 0 \\
-\sin \phi & \cos \phi & 0 \\
0 & 0 & 1
\end{array}\right]\left[\begin{array}{c}
\dot{\psi} \\
\dot{\theta} \\
\dot{\phi}
\end{array}\right],}
\end{aligned}
$$

where $u, v$, and $w$ denote the components of the rotor velocity along the $x_{f^{-}}, y_{f^{-}}$, and $z_{f}$-axes, respectively.

3.2. Equation Motion of Radial Force for AMB System. The rotor was assumed to be a rigid body and $\left(x_{f}, y_{f}, z_{f}\right)$ was assumed to be fixed to the end of the shaft and rotate with it, as indicated in Figure 6. Therefore, the angular velocity of $\left(x_{r}\right.$, $\left.y_{r}, z_{r}\right)$ is equal to that of the rotor, and it follows that [22]

$$
\begin{aligned}
\vec{\omega}_{x y z} & =p \vec{i}+q \vec{j}+r \vec{k}, \\
\vec{H}_{0} & =J_{x} p \vec{i}+J_{y} q \vec{j}+J_{z} r \vec{k},
\end{aligned}
$$

where $\vec{\omega}_{x y z}$ are the angular velocities of the coordinate system $\left(x_{r}, y_{r}, z_{r}\right), \vec{H}_{0}$ is the angular momentum around the origin $O$, and $J_{x}, J_{y}, J_{z}$ are the moments of inertia around the $x_{r^{-}}$, $y_{r^{-}}$, and $z_{r^{-}}$axes, respectively. Calculating (8) with respect to time yields

$$
\begin{aligned}
\dot{\vec{H}}_{0}= & \left(\dot{\vec{H}}_{0}\right)_{x y z}+\vec{\omega}_{x y z} \vec{H}_{0} \\
= & {\left[J_{x} \dot{p}-\left(J_{y}-J_{z}\right) q r\right] \vec{i}+\left[J_{x} \dot{q}+\left(J_{y}-J_{z}\right) p r\right] \vec{j} } \\
& +\left[J_{z} \dot{r}+\left(J_{y}-J_{x}\right)\right] \vec{k} .
\end{aligned}
$$


Assuming that the rotor is a rigid body and symmetrical with respect to the $z_{r}$-axis and that $J_{x}=J_{y}$, the following equations of motion are obtained:

$$
\begin{array}{r}
J_{x} \dot{p}-\left(J_{y}-J_{z}\right) q r=M, \\
J_{x} \dot{q}+\left(J_{y}-J_{z}\right) p r=N, \\
J_{z} \dot{r}=L .
\end{array}
$$

Figure 6 presents a coordinate system of moving frame $G x_{r} y_{r} z_{r}$ from fixed frame $(O)$ to the magnetic bearing. The angular velocity components of the rotor along the $x_{r^{-}}, y_{r^{-}}$, and $z_{r}$-axes are denoted by $p, q$, and $r$, respectively. The moments of external force along $x_{r^{-}}, y_{r^{-}}$, and $z_{r^{-}}$-axes are denoted by $M, N$, and $L$, respectively. In physical terms, $p$, $q$, and $r$ represent the angular velocities of pitching, yawing, and rolling, respectively (Figure 6).

The rotor, suspended by four magnetic forces, is attached to a shaft that is connected to a fixed driving motor with a coupler on the other side (Figure 4). Thus the external torques exerted on the rotor can be described as

$$
\begin{aligned}
& x_{r} \text {-axis: }\left(f_{1 x}-f_{2 x}-\alpha_{o}\right) l, \\
& y_{r} \text {-axis: }\left(f_{1 y}-f_{2 y}-M g-\beta_{o}\right) l, \\
& z_{r} \text {-axis: } T_{m}-\rho r-T_{0},
\end{aligned}
$$

where $\alpha_{o}$ and $\beta_{o}$ are the damping and stiffness force exerted by the coupler, $T_{m}$ is the motor torque, $\rho$ is the damping torque coefficient, and $T_{0}$ is the coulomb friction torque. Assuming that the rotor has a small displacement $x_{r}$ and $y_{r}$ in the horizontal and vertical planes, respectively, $\alpha_{o}$ and $\beta_{o}$ are given by

$$
\begin{aligned}
& \alpha_{o}=\frac{k l_{1}}{l} x_{o}+\frac{c l_{2}}{l} \dot{x}_{o} \\
& \beta_{o}=\frac{k l_{1}}{l} y_{o}+\frac{c l_{2}}{l} \dot{y}_{o},
\end{aligned}
$$

where $c$ and $k$ are displacement coefficients and $l_{1}$ and $l_{2}$ are the distances between the shaft center and the flexible coupling and the magnetic bearing, respectively. By using (4), the forces exerted on the rotor and the moments around the original point are represented by the following matrix forms in (15) and (16), respectively. Consider

$$
\begin{aligned}
& {\left[\begin{array}{l}
x_{r} \\
y_{r}
\end{array}\right]} \\
& =\left[\begin{array}{cc}
\cos \phi & \sin \phi \\
-\sin \phi & \cos \phi
\end{array}\right]\left[\begin{array}{c}
f_{1 x}-f_{2 x}-\alpha_{o} \\
f_{1 y}-f_{2 y}-M g-\beta_{o}
\end{array}\right], \\
& {\left[\begin{array}{c}
M \\
N
\end{array}\right]} \\
& =\left[\begin{array}{cc}
\cos \phi & \sin \phi \\
-\sin \phi & \cos \phi
\end{array}\right]\left[\begin{array}{c}
-\left(\begin{array}{c}
f_{1 y}-f_{2 y}-M g-\beta_{o} \\
\left(f_{1 x}-f_{2 x}-\alpha_{o}\right) l
\end{array}\right] l
\end{array} .\right.
\end{aligned}
$$

Deriving (6) leads to

$$
\left[\begin{array}{c}
\dot{p} \\
\dot{q}
\end{array}\right]=\dot{\phi}\left[\begin{array}{cc}
-\sin \phi & \cos \phi \\
-\sin \phi & -\cos \phi
\end{array}\right]+\left[\begin{array}{cc}
\cos \phi & \sin \phi \\
-\sin \phi & \cos \phi
\end{array}\right]\left[\begin{array}{c}
\ddot{\theta} \\
\ddot{\psi}
\end{array}\right] .
$$

Equation (17) can be rearranged to

$$
\left[\begin{array}{c}
\ddot{\theta} \\
\ddot{\psi}
\end{array}\right]=\left[\begin{array}{cc}
\cos \phi & -\sin \phi \\
\sin \phi & \cos \phi
\end{array}\right]\left[\begin{array}{c}
\dot{p} \\
\dot{q}
\end{array}\right]-r\left[\begin{array}{cc}
0 & 1 \\
-1 & 0
\end{array}\right]\left[\begin{array}{l}
\dot{\theta} \\
\dot{\psi}
\end{array}\right] .
$$

By using (10) and (11), (18) becomes

$$
\begin{aligned}
{\left[\begin{array}{c}
\ddot{\theta} \\
\ddot{\psi}
\end{array}\right]=} & \frac{1}{J_{x}}\left[\begin{array}{cc}
\cos \phi & -\sin \phi \\
\sin \phi & \cos \phi
\end{array}\right]\left[\begin{array}{l}
M \\
N
\end{array}\right] \\
& +\frac{\left(J_{x}-J_{z}\right) r}{J_{x}} \times\left[\begin{array}{cc}
\cos \phi & -\sin \phi \\
\sin \phi & \cos \phi
\end{array}\right]\left[\begin{array}{c}
q \\
-p
\end{array}\right] \\
& -r\left[\begin{array}{cc}
0 & 1 \\
-1 & 0
\end{array}\right]\left[\begin{array}{l}
\dot{\theta} \\
\dot{\psi}
\end{array}\right] .
\end{aligned}
$$

Using (14), by letting $x_{1}=l \psi$ and $y_{1}=-l \theta$ and eliminating $p$ and $q$ in (19) and assuming $\psi$ and $\theta$ to be small, (19) becomes a linearizing equation of motion as follows:

$$
\begin{aligned}
{\left[\begin{array}{c}
\ddot{\theta} \\
\ddot{\psi}
\end{array}\right]=} & {\left[\begin{array}{cc}
\frac{-c l l_{1}}{J_{x}} & \frac{-r J_{z}}{J_{x}} \\
\frac{r J_{z}}{J_{x}} & \frac{-c l l_{1}}{J_{x}}
\end{array}\right]\left[\begin{array}{l}
\dot{\theta} \\
\dot{\psi}
\end{array}\right]-\frac{k l l_{1}}{J_{x}}\left[\begin{array}{ll}
1 & 0 \\
0 & 1
\end{array}\right]\left[\begin{array}{l}
\theta \\
\psi
\end{array}\right] } \\
& +\frac{l}{J_{x}}\left[\begin{array}{c}
-f_{1 y}+f_{2 y}+M g \\
f_{1 x}-f_{2 x}
\end{array}\right] .
\end{aligned}
$$

From (20), the block diagram can be drawn, as indicated in Figure 7. In this figure, the motion along the $x_{f}$-axis and the $y_{f}$-axis is independent if $r=0$. The horizontal motion is coupled with the vertical motion if $r \neq 0$. This is the so-called gyroscopic effect. Furthermore, the coupling effect increases with $r$. To decrease the interaction between the $x$ - and $y$-axes, the $J_{z} / J_{x}$ ratio should be decreased [23].

\section{Neural Fuzzy Controller Design for Position Control}

4.1. Fuzzy Logic Controller (FLC). Recently, the FLC has emerged as an effective tool for stabilizing a nonlinear system, such as an AMB system, a magnetic levitation system, or other electronic devices [24-30]. The FLC is a methodical approach for controlling a nonlinear system and is a heuristic technique for enhancing the operation of a closed loop system. Although FLC performance is a function of its ability to simulate many functions simultaneously, its output results are considerably thorough. The FLC presented in Figure 8 is composed of four main parts: one performs fuzzification; one provides the rule base; one is an inference engine; one performs defuzzification.

The structure of a fuzzy controller, based on RBFNN identification of the AMB system, is presented in Figure 9. It consists of a fuzzy controller, a reference model, an RBFNN, 


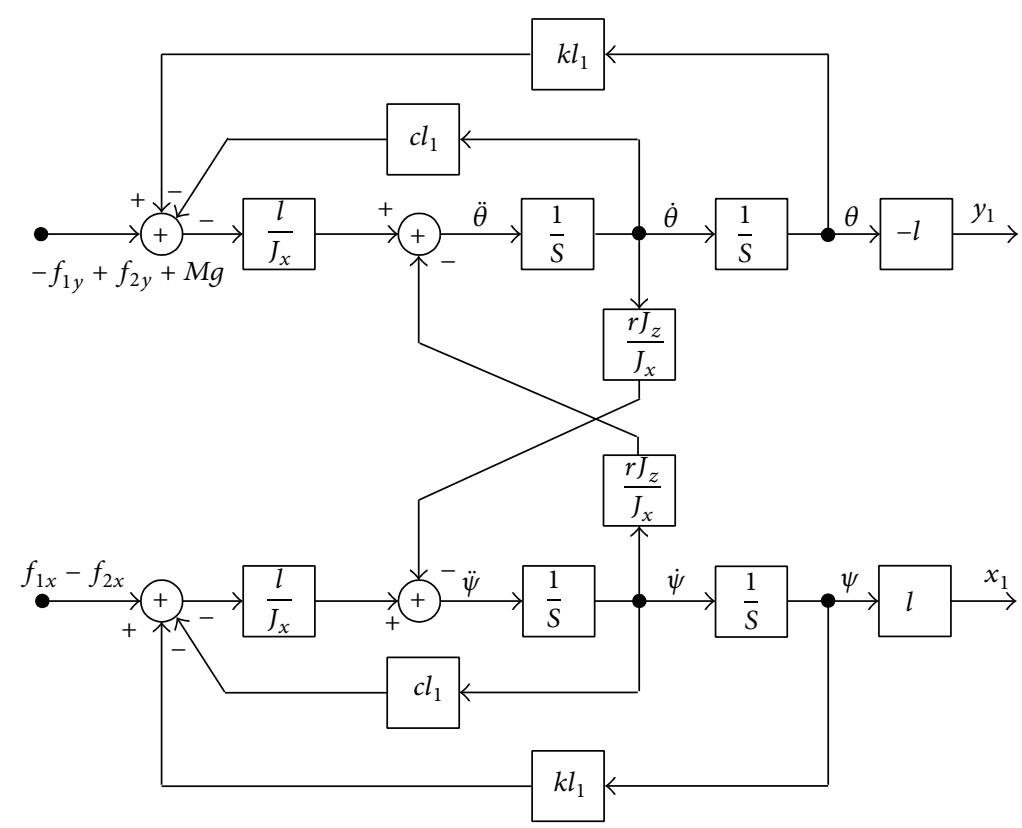

FIGURE 7: Diagram radial of pitching, yawing, and rolling of rotor.

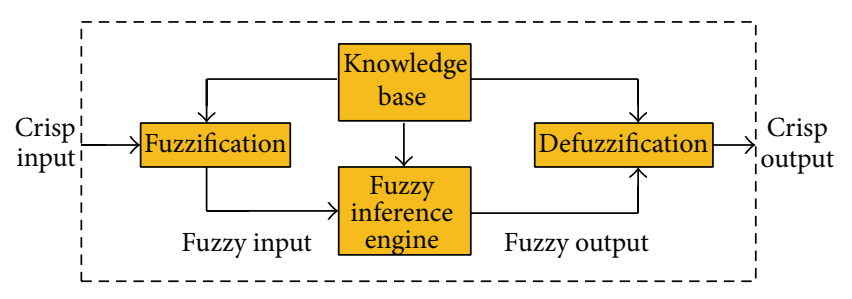

Figure 8: Fuzzy logic controller block.

and an adjusting mechanism. The operating principle of this controller is based on AMB dynamics and control knowledge can be incorporated into an NFC design based on RBFNN identification [14, 31, 32]. A detailed description of these is shown in Figure 9, where $x^{*}$ and $x_{r m}$ are a step reference and a reference model, respectively. $x_{r b f}$ and $u_{\mathrm{fn}}$ are the outputs of neural and fuzzy neural controller, respectively. $x_{p}$ is the response of displacement; $Z^{-1}$ is the back shift operator. $u_{f}$ represents the output of the fuzzy controller. FPID is a fuzzy proportional integral derivative control for the current loop [33]. $F_{d}$ is the external torque from ventilator. FI and DFI are the fuzzification converted controller inputs ( $e$ and $d e$ ), respectively, and the defuzzification based on product inference rule center average defuzzification the conclusions of the inference mechanism into actual inputs.

Based on Figure 9, the tracking error $e$ and the error change $d e$ are defined by the following equations:

$$
\begin{aligned}
e(k) & =x_{r m}(k)-x_{p}(k) \\
d e(k) & =e(k)-e(k-1) .
\end{aligned}
$$

The design procedure of the fuzzy controller algorithm is as follows. First, $e$ and $d e$ are taken as the input variables of the fuzzy controller, and their linguistic variables are defined as $E$ and $d E$. The linguist values of $E$ and $d E$ are $\left\{A_{0}, A_{1}, A_{2}, A_{3}, A_{4}, A_{5}, A_{6}\right\}$ and $\left\{B_{0}, B_{1}, B_{2}, B_{3}, B_{4}, B_{5}, B_{6}\right\}$, respectively. Each linguistic value of $E$ and $d E$ is based on the symmetrical triangular membership function, which is presented in Figure 10. Second, the membership degrees of $e$ and $d e$ are computed. Figure 10 indicates that only two linguistic values are excited in any input value, and it is the membership degree. The variables $\mu_{A_{i}}(e)$ and $\mu_{B_{j}}(d e)$ are easily derived using the flowchart presented in the figure, and the membership degree is obtained as follows [34]:

$$
\begin{aligned}
\mu_{A_{i}}(e) & =\frac{e_{i+1}-e}{e_{i+1}-e_{i}}, \\
\mu_{A_{i+1}}(e) & =1-\mu_{A_{i}}(e) .
\end{aligned}
$$

Similar results are obtained on computing the membership degree $\mu_{B_{j}}(d e)$. Third, the initial fuzzy controller rules are selected by referring to the dynamic response characteristics, such as

$$
\text { IF } e \text { is } A_{i} \text { and } d e \text { is } B_{j} \text { THEN } u_{f} \text { is } c_{j, i},
$$

where $i$ and $j$ range from 0 to $6, A_{i}$ and $B_{j}$ are fuzzy numbers, and $c_{j, i}$ is the real number. The graph of fuzzification and the fuzzy rule table is presented in Figure 10. Finally, the fuzzy system $u_{f}(e, d e)$ is constructed using the singleton fuzzifier, product inference rule, and the central average defuzzifier method is presented. Although 49 fuzzy rules which are listed in Figure 10 will be inferred, only four fuzzy rules can be effectively excited to generate a nonzero output. Therefore, if an error $e$ is located between $e_{i}$ and $e_{i+1}$ and an error change $d e$ is located between $d e_{j}$ and $d e_{j+1}$, only four linguistic values $A_{i}, A_{i+1}, B_{j}$, and $B_{j+1}$ and the corresponding consequent 


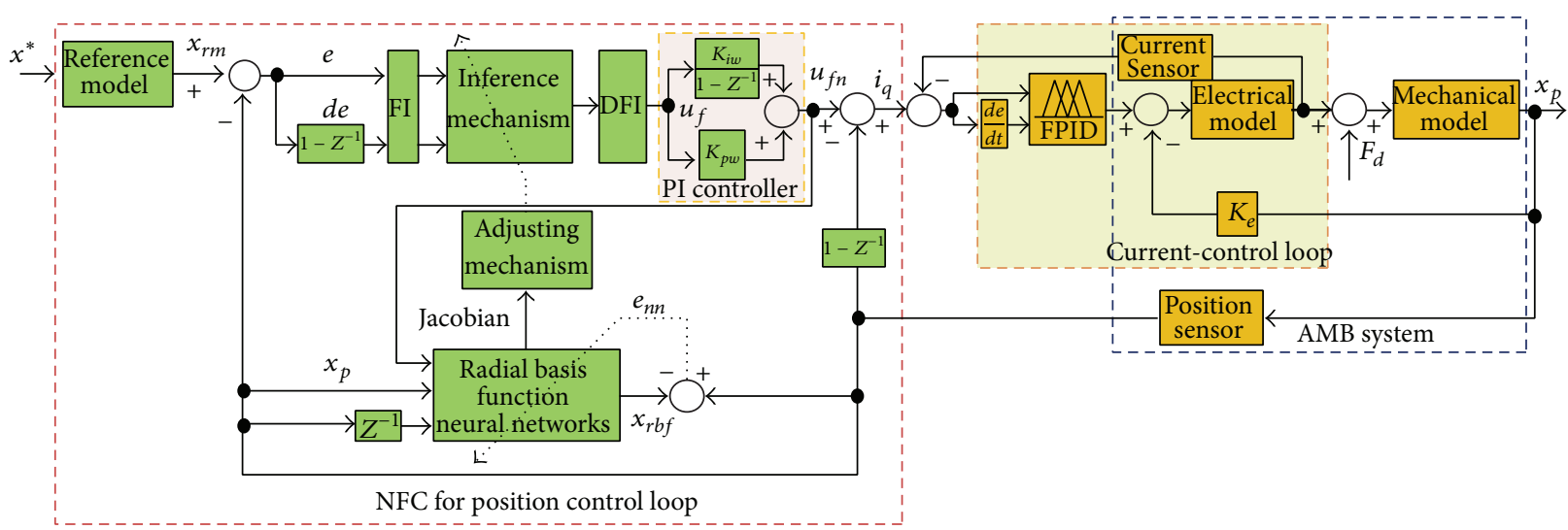

FIGURE 9: Block diagram neural fuzzy control for AMB system.

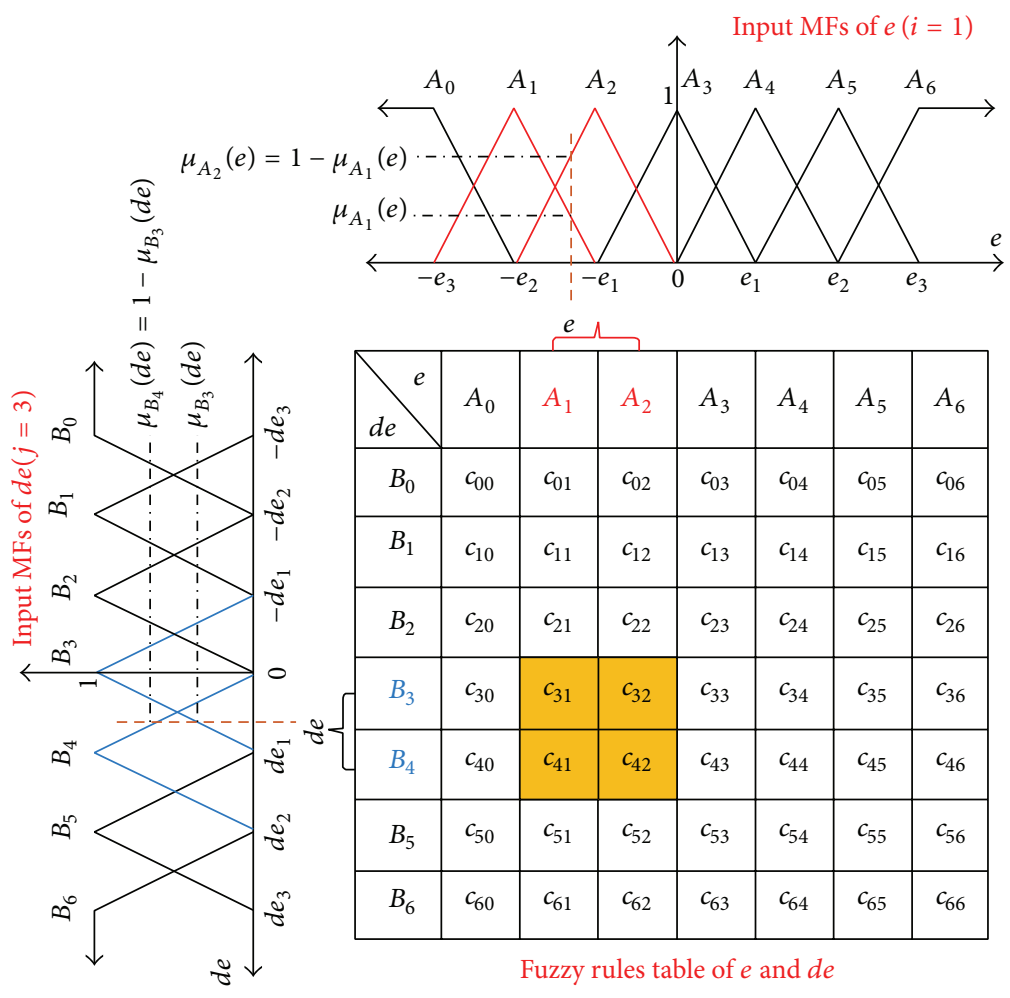

Figure 10: The membership functions of $e, d e$, and fuzzy rule table.

values $c_{j, i}, c_{j+1, i}, c_{j, i+1}$, and $c_{j+1, i+1}$ can be excited, and the output of the fuzzy controller can be calculated using the following equation:

$$
\begin{aligned}
u_{f}(e, d e) & =\frac{\sum_{n=i}^{i+1} \sum_{m=j}^{j+1} c_{m, n}\left[\mu_{A_{n}}(e) \times \mu_{B_{m}}(d e)\right]}{\sum_{n=i}^{i+1} \sum_{m=j}^{j+1} \mu_{A_{n}}(e) \times \mu_{B_{m}}(d e)} \\
& =\sum_{n=i}^{i+1} \sum_{m=j}^{j+1} c_{m, n} \times d_{n, m},
\end{aligned}
$$

where $d_{n, m}=\mu_{A_{n}}(e) \times \mu_{B_{m}}(d e)$. And those $c_{m, n}$ are adjustable parameters for fuzzy controller. In addition, by using (22), the value of $\sum_{n=i}^{i+1} \sum_{m=j}^{j+1} d_{n, m}=1$ in (24) can be easily derived. The $u_{f n}$ is formulated by the output of fuzzy controller $\left(u_{f}\right)$ and the output of the integral (I) controller $\left(u_{f n i}\right)$ as follows:

$$
\begin{aligned}
u_{f n}(k) & =u_{f n i}(k-1)+\left(K_{p w}+K_{i w}\right) \times u_{f}(k) \\
\Delta u_{f n}(k) & =u_{f n}(k)-u_{f n}(k-1) .
\end{aligned}
$$

\subsection{Radial Basic Function Neural Network}

4.2.1. Radial Basis Function Neural Network Architecture. The RBFNN adopted in this part of the study was a three-layer structure, as shown in Figure 11 and comprised an input layer, 


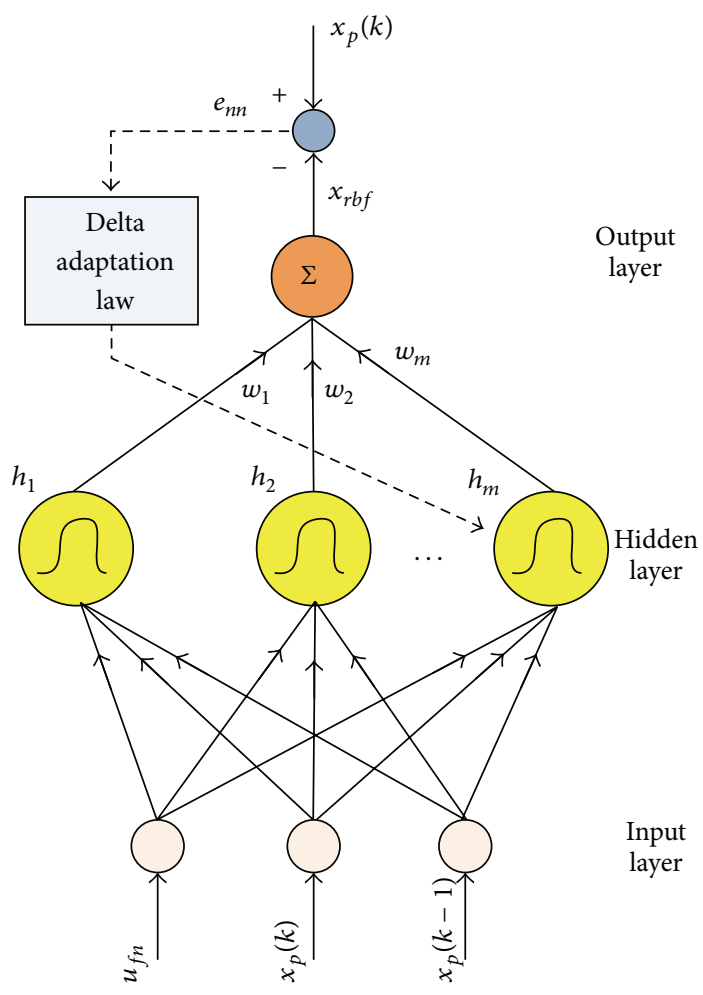

FIGURE 11: The architecture of RBFNN.

a hidden layer, and an output layer. Each layer is explained in detail below.

Input Layer. the RBFNN has three inputs by $u_{f n}(k), x_{p}(k)$, $x_{p}(k-1)$ and its vector form is represented by

$$
X=\left[u_{f n}(k), x_{p}(k), x_{p}(k-1)\right]^{T} .
$$

Hidden Layer. The hidden layer is composed of $n$ RBFNNs that are connected directly to all the elements in the output layer. A node in the hidden layer produces a greater output when the input pattern presented is closer to its center. The multivariate Gaussian function is used as the activated function in the hidden layer of the RBFNN, which is given by the following equations:

$$
h_{j}(X)=e^{-(1 / 2)\left\|X-c_{j}\right\|^{2} / b_{j}^{2}}, \quad j=1,2,3, \ldots, m,
$$

where $b_{j}$ denotes the node center and node variance of the $j$ th neuron, $m$ is the number of neurons in the hidden layer, $\left\|X-c_{\mathbf{j}}\right\|$ is the norm value which is measured by the inputs and the node center at each neuron, and $\mathbf{c}_{\mathbf{j}}=\left[c_{j 1}, c_{j 2}, \ldots, c_{j n}\right]^{T}$ is the center of the $j$ th RBFNN unit.

Output Layer. The network output $x_{r b f}$ is formed by a linearly weighted sum of the number of basic functions in the hidden layer. The network output in Figure 11 is derived using the following equation:

$$
x_{r b f}=\sum_{j=1}^{m} w_{j} h_{j},
$$

where $w_{j}$ and $h_{j}$ are the weights from the $j$ th hidden layer neuron to the output layer neuron and the output of the $j$ th node in the hidden layer, respectively.

\subsubsection{Training Algorithm in Radial Basis Function Neural} Network. To describe the online learning algorithm of the proposed NFC, the energy function $E$ is defined as follows:

$$
E=\frac{1}{2}\left(x_{p}-x_{r b f}\right)^{2}=\frac{1}{2} e_{n n}^{2} .
$$

Based on the gradient descent method, the learning algorithm of the weights, node center, and variance are adjusted using the following equations:

$$
\begin{aligned}
& w_{j}(k+1) \\
& \quad=w_{j}(k)+\eta e_{n n}(k) h_{j}(k) \\
& c_{j i}(k+1) \\
& \quad=c_{j i}(k)+\eta e_{n n}(k) w_{j}(k) h_{j}(k) \frac{X_{i}(k)-c_{j i}(k)}{b_{j}^{2}(k)} \\
& b_{j}(k+1) \\
& \quad=b_{j}(k)+\eta e_{n n}(k) w_{j}(k) h_{j}(k) \frac{\left\|X_{i}(k)-c_{j i}(k)\right\|^{2}}{b_{j}^{3}(k)},
\end{aligned}
$$

where $j=1,2, \ldots, m, i=1,2,3$, and $\eta$ is a learning rate. Further, $\partial x_{p} / \partial u_{f n}$ is Jacobian transformation and can 
be derived from Figure 11 and (27) and (28). Consider the following:

$$
\frac{\partial x_{p}}{\partial u_{f n}} \approx \frac{\partial x_{r b f}}{\partial u_{f n}}=\sum_{j=1}^{m} w_{j} h_{j} \frac{c_{j 1}-u_{f n}(k)}{b_{j}^{2}} .
$$

4.3. Reference Model. A second order system with natural frequency $\omega_{n}$ and damping ratio $\varsigma$ is used as a reference model in the adaptive control system, as explained in the following equation:

$$
\frac{x_{r m}(s)}{x^{*}(s)}=\frac{\omega_{n}^{2}}{s^{2}+2 \varsigma \omega_{n}+\omega_{n}^{2}} .
$$

By applying the bilinear transformation, the second order system (32) can be transformed into a discrete model using

$$
\frac{\omega_{r}\left(z^{-1}\right)}{\omega_{r}^{*}\left(z^{-1}\right)}=\frac{a_{0}+a_{1} z^{-1}+a_{2} z^{-2}}{1+b_{1} z^{-1}+b_{2} z^{-2}} .
$$

Furthermore, the difference equation is written as follows:

$$
\begin{aligned}
\omega_{r}(k)= & -b_{1} \omega_{r}(k-1)-b_{2} \omega_{r}(k-2)+a_{0} \omega_{r}^{*}(k) \\
& +a_{1} \omega_{r}^{*}(k-1)+a_{2} \omega_{r}^{*}(k-2) .
\end{aligned}
$$

4.4. Fuzzy Logic Control Parameters Adjusting Mechanism. The gradient descent method is used to derive the FLC control law in Figure 9. The adjusting parameters of the fuzzy controller minimize the square error between the rotor displacement and the output of the reference model. The instantaneous cost function is defined as follows:

$$
E_{e}=\frac{1}{2} e^{2}=\frac{1}{2}\left(x_{r m}-x_{p}\right)^{2} .
$$

The parameters of $c_{m, n}$ are adjusted according to

$$
\Delta c_{m, n}=-\alpha \frac{\partial E_{e}}{\partial c_{m, n}},
$$

where $\alpha$ represents the adaptive rate of the system. The chain rule is used, and the partial differential equation for $E_{e}$ in (35) is written as follows:

$$
\frac{\partial E_{e}}{\partial c_{m, n}}=-e \frac{\partial x_{p}}{\partial u_{f}} \frac{\partial u_{f}}{\partial c_{m, n}} .
$$

From (24) and using the Jacobian formulation from (31), the following equation is derived:

$$
\frac{\partial u_{f}(k)}{\partial c_{m, n}(k)}=d_{n, m}
$$

It is difficult to calculate $\partial x_{p} / \partial u_{f}$ because of unknown plant dynamics. To overcome this problem and to increase the online learning rate of the connective weights, a delta adaptation law is proposed as follows:

$$
\begin{aligned}
\frac{\partial x_{p}}{\partial u_{f}} & \approx\left(K_{p w}+K_{i w}\right) \frac{\partial x_{r b f}}{\partial u_{f n}} \\
& =\left(K_{p w}+K_{i w}\right) \sum_{j=1}^{m} w_{j} h_{j} \frac{c_{j 1}-u_{f n}(k)}{b_{j}^{2}},
\end{aligned}
$$

where $K_{p w}$ and $K_{i w}$ are the PI controller gains. Therefore, substituting (38) and (39) into (37) and combining with (36), the parameter $c_{m, n}$ of the fuzzy controller described in (24) can be adjusted using $m=j, j+1$, and $n=i, i+1$, as indicated by the following equation:

$$
\begin{aligned}
& \Delta c_{m, n}(k) \\
& \quad=\alpha e(k)\left(K_{p w}+K_{i w}\right) d_{n, m} \sum_{j=1}^{m} w_{j} h_{j} \frac{c_{j 1}-u_{f n}(k)}{b_{j}^{2}} .
\end{aligned}
$$

\section{Stability Analysis for Active Magnetic Bearing System}

The stability of the controller of an AMB system is critical to its functioning, particularly during start-up. After the operation of the controller starts, the rotor remains on touchdown surfaces. While the rotor stays at the position, the controller can never estimate the motion of the rotor inside the stator because the electromotive force is proportional to the rotational speed. Hence, movement toward its operating position, the center of the two pair of electromagnets, is necessary for the convergence of controller target. To ensure stable functioning of the system, a stability analysis of the NFC was conducted [35-37]. When the parameters of an NFC move toward infinity, the NFC becomes an unstable controller of the AMB system; when the center of one of the membership functions (MFs) approaches infinity, the NFC is stable. Because the firing of the rule which contains these MFs is equal to zero, other rules can identify the system without instability and when the standard deviations of MFs move toward infinity the values of the MFs are equal to one, and the output is finite; therefore, the areas of MFs that are optimal for the NFC to control an AMB system must be used. The RBFNN adopted in this study comprises three inputs, as indicated in (26). The output is represented by (24), and the objective function is defined by (35). The consequent weights are as follows:

$$
W=\left[\begin{array}{lll}
\vec{W}_{1} & \vec{W}_{2} & \vec{W}_{3}, \ldots, \vec{W}_{n}
\end{array}\right]_{m \times(n+1)} .
$$

Two types of parameter are defined as the antecedent parameters. The first type is the means of the MFs as follows:

$$
F=\left[\begin{array}{lll}
\vec{F}_{1}^{T} & \vec{F}_{2}^{T} & \vec{F}_{3}^{T}, \ldots, \vec{F}_{n}^{T}
\end{array}\right] .
$$

The second type is the standard deviations of the MFs as follows:

$$
S=\left[\begin{array}{lll}
\vec{S}_{1}^{T} & \vec{S}_{2}^{T} & \vec{S}_{3}^{T}, \ldots, \vec{S}_{n}^{T}
\end{array}\right]
$$

From (29), the discrete Lyapunov function is defined using (44) as follows:

$$
V(k)=E(k)=\frac{1}{2} e^{2}(k)=\frac{1}{2}\left(x_{p}(k)-x_{r b f}(k)\right)^{2} .
$$


The change of the Lyapunov function at each iteration of (44) is obtained using

$$
\begin{aligned}
\Delta V(k) & =V(k+1)-V(k)=\frac{1}{2}\left[e^{2}(k+1)-e^{2}(k)\right] \\
& =\frac{1}{2}[e(k+1)-e(k)][e(k+1)+e(k)] \\
& =\Delta e(k)\left[\frac{1}{2} \Delta e(k)+e(k)\right] .
\end{aligned}
$$

Equations (41) to (45) were applied to achieve an approximate error modification for the NFC as shown in the following equation:

$$
\Delta e(k)=\theta(k)+\vartheta(k)+\rho(k)
$$

with

$$
\begin{aligned}
& \theta(k)=\left(\frac{\partial e(k)}{\partial S(k)}\right)^{T} \Delta S(k), \\
& \vartheta(k)=\left(\frac{\partial e(k)}{\partial F(k)}\right)^{T} \Delta F(k), \\
& \rho(k)=\operatorname{tr}\left(\left(\frac{\partial e(k)}{\partial W(k)}\right)^{T} \Delta W(k)\right),
\end{aligned}
$$

where the $\operatorname{tr}(\cdot)$ is the trace of matrices. In this controller, only train the consequent parameters so $\theta(k)=0 ; \vartheta(k)=0$. Therefore, substituting (46) and (47) into (45) obtains

$$
\begin{aligned}
\Delta V(k)= & \rho(k)\left[\frac{\rho(k)}{2}+e(k)\right] \\
= & \operatorname{tr}\left(\left(\frac{\partial e(k)}{\partial W(k)}\right)^{T} \Delta W(k)\right) \\
& \times\left[\frac{1}{2} \operatorname{tr}\left(\left(\frac{\partial e(k)}{\partial W(k)}\right)^{T} \Delta W(k)\right)+e(k)\right] .
\end{aligned}
$$

The updating rule for conclusion parameters is

$$
\begin{aligned}
\frac{\partial e(k)}{\partial W(k)} & =\frac{\partial e(k)}{\partial x_{r b f}(k)} \times \frac{\partial x_{r b f}(k)}{\partial W(k)}=-\frac{\partial x_{r b f}(k)}{\partial W(k)}, \\
\Delta W(k) & =\eta e(k) \frac{\partial x_{r b f}(k)}{\partial W(k)},
\end{aligned}
$$

where $\eta$ is the learning rate that is used to adjust consequent parameters. From (48) and (49) can be rewriting as follows:

$$
\begin{aligned}
\Delta V(k)= & -\eta e^{2}(k)\left(\left\|\frac{\partial x_{r b f}(k)}{\partial W(k)}\right\|_{F}\right)^{2} \\
& +\frac{1}{2} \eta^{2} e^{2}(k)\left(\left\|\frac{\partial x_{r b f}(k)}{\partial W(k)}\right\|_{F}\right)^{4},
\end{aligned}
$$

where $\|\cdot\|_{F}$ is the Frobenius norm, assuming that

$$
\begin{aligned}
\Psi_{W}(k) & =\frac{\partial x_{r b f}(k)}{\partial W(k)}, \\
\Psi_{W^{\max }} & =\max _{k}\left\|\Psi_{W}(k)\right\|_{F}, \\
\Upsilon_{W} & =\frac{1}{2} \eta\left(\left\|\Psi_{W}(k)\right\|_{F}\right)^{2}\left(2-\eta\left(\left\|\Psi_{W}(k)\right\|_{F}\right)^{2}\right) .
\end{aligned}
$$

According to the standard Lyapunov theory, the change of the Lyapunov function must be less than zero to ensure stability. This can be achieved using (50) and (51) as follows:

$$
\begin{aligned}
\Delta V(k) & <0, \\
\Delta V(k) & =-\Upsilon_{W} e^{2}(k) \Longrightarrow \Upsilon_{W}>0, \\
0 & <\eta<\frac{2}{\left(\left\|\Psi_{W}(k)\right\|_{F}\right)^{2}} .
\end{aligned}
$$

Equation (52) is an adaptive constraint and the learning rate stability condition changes at each iteration. Therefore, this constraint can be used easily for online training. By calculating (52), the following equation can be derived:

$$
0<\eta<\frac{2}{\left(\Psi_{W}^{\max }\right)^{2}}
$$

with $\Psi_{W}^{\max }=\max _{k}\left\|\Psi_{W}(k)\right\|_{F}$.

Equation (53) is a conservative constraint and cannot be calculated through online identification. The equation can also be written using the chain rule as follows:

$$
\begin{aligned}
\rho(k) & =\operatorname{tr}\left(\left(\frac{\partial e(k)}{\partial W(k)}\right)^{T} \Delta W(k)\right) \\
& =\eta e(k)\left(\left\|\Psi_{W}(k)\right\|_{F}\right)^{2} .
\end{aligned}
$$

The learning rate of the consequent part is selected to satisfy (54) so that the identifier is stable at each learning cycle. The adaptive controller functions online during AMB system operation. The results of the AMB system are presented in the next section.

\section{Results and Discussions}

The experimental setup of this study is presented in Figure 12. The laboratory setup included a horizontal shaft magnetic bearing that was symmetrical and was controlled by two axes.

The system was driven by an induction motor with a flexible coupling to isolate the motor vibration. The magnetic bearing included four identical electromagnets that were equally spaced radially around a rotor composed of laminated stainless steel, as indicated in Figure 13. Each electromagnet included a coil and a laminated core composed of silicon steel. The rotor displacement along the vertical $y$ - and horizontal $x$-axes of the geometric center of the shaft was measured using a pair of eddy current sensors, as indicated in Figure 14. The conversion time of the 16-bit A/D converter was $10 \mu \mathrm{s}$. 


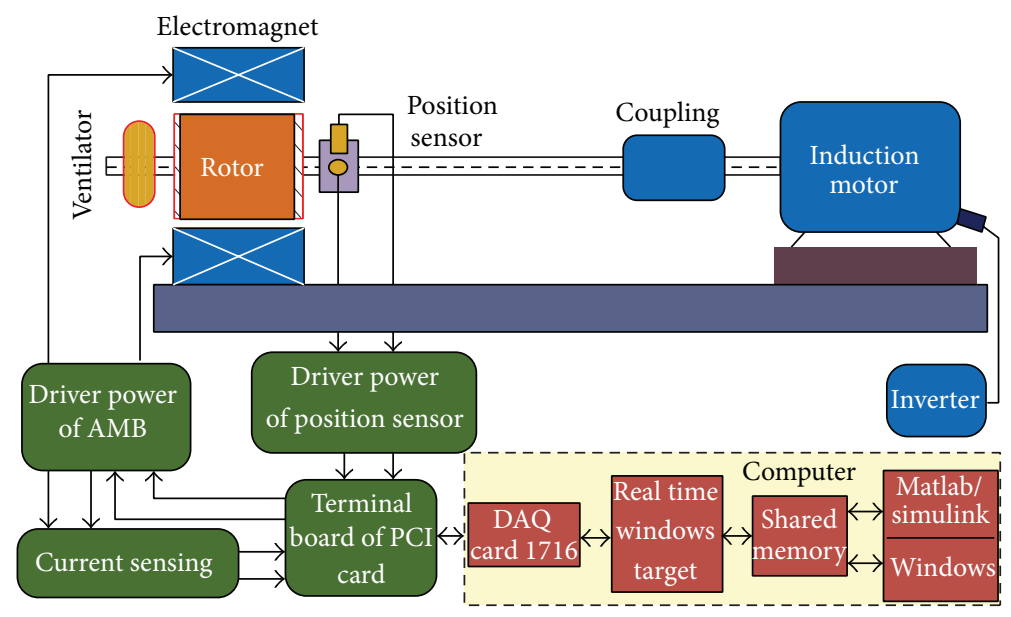

FIgURE 12: The experimental setup of the AMB system.

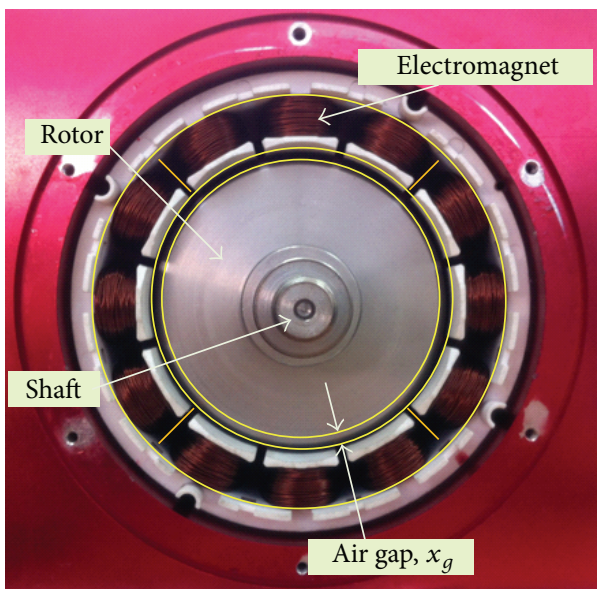

(a)

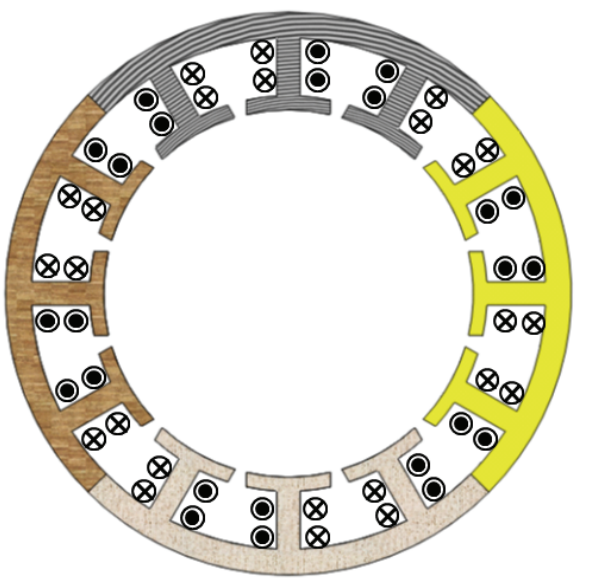

(b)

Figure 13: Inside view (a) and layout (b) of magnetic bearing.
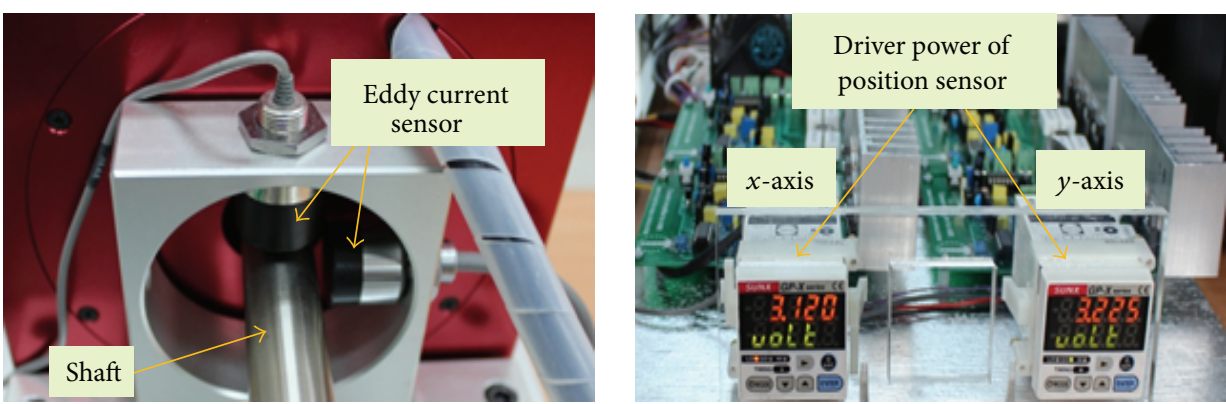

FIGURE 14: Eddy sensor and driver power. 


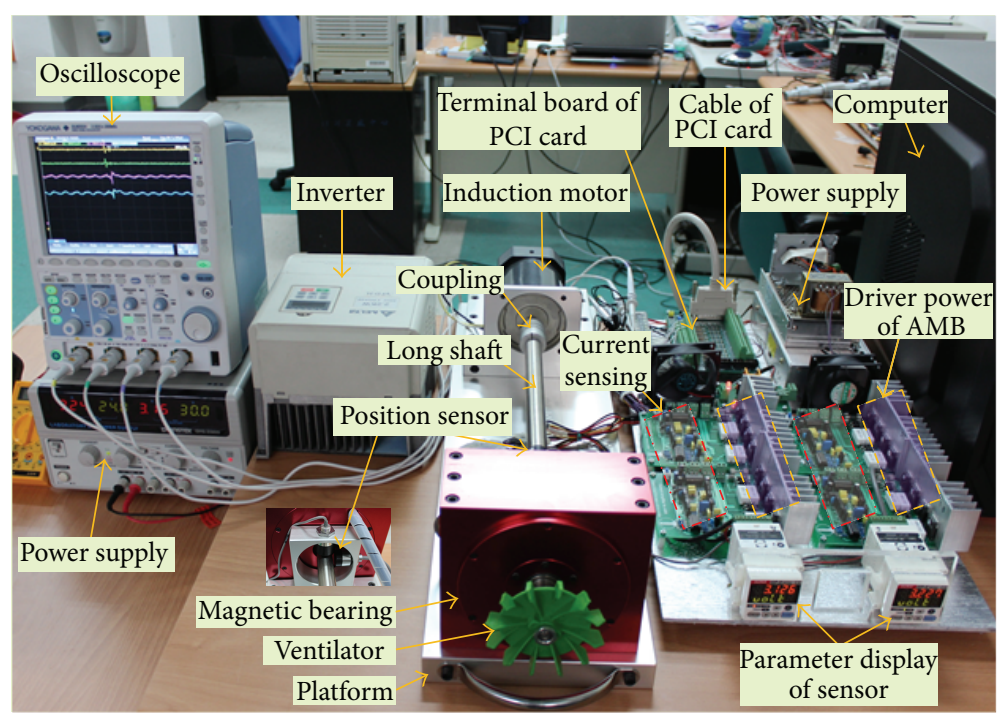

FIGURE 15: Photograph of the experimental setup.

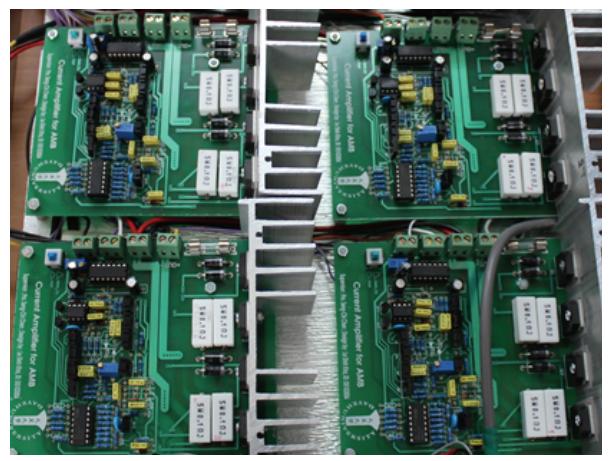

FIgURE 16: Photograph of current amplifier and current sensing.

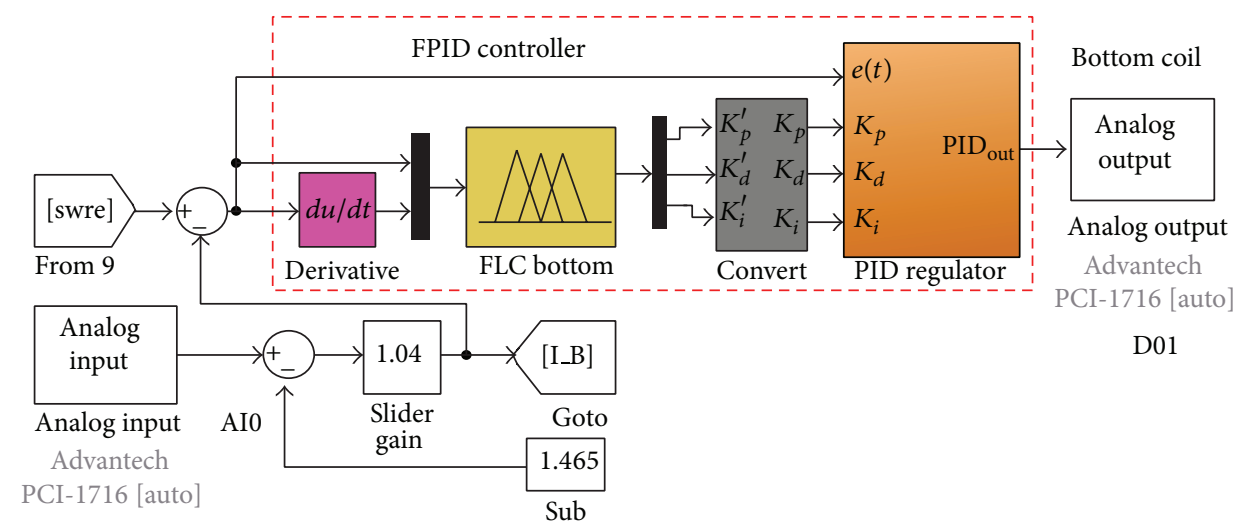

FIGURE 17: Model of real time window target for current-control loop. 


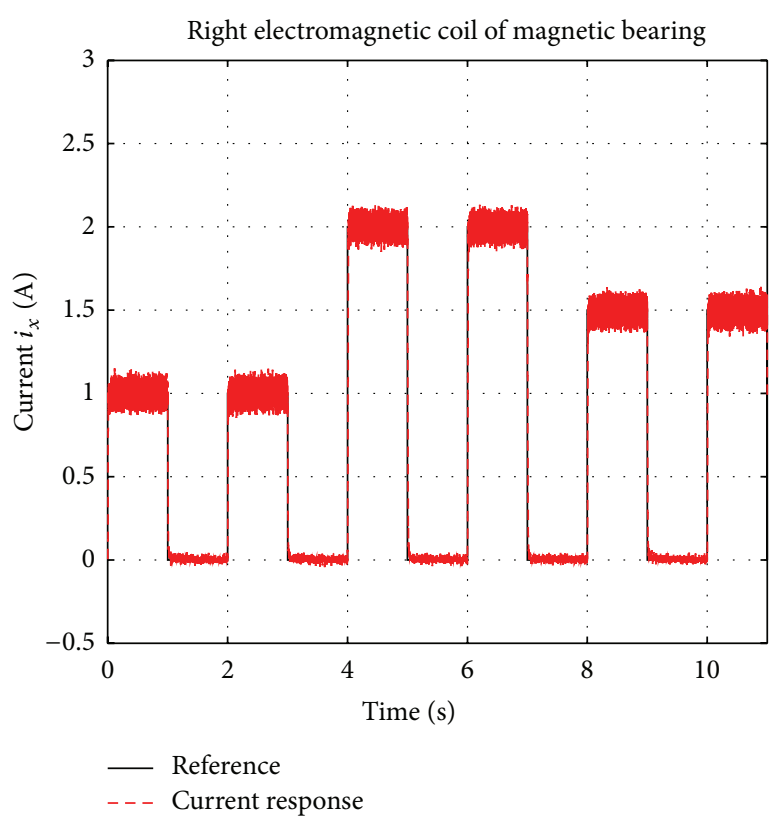

(a)

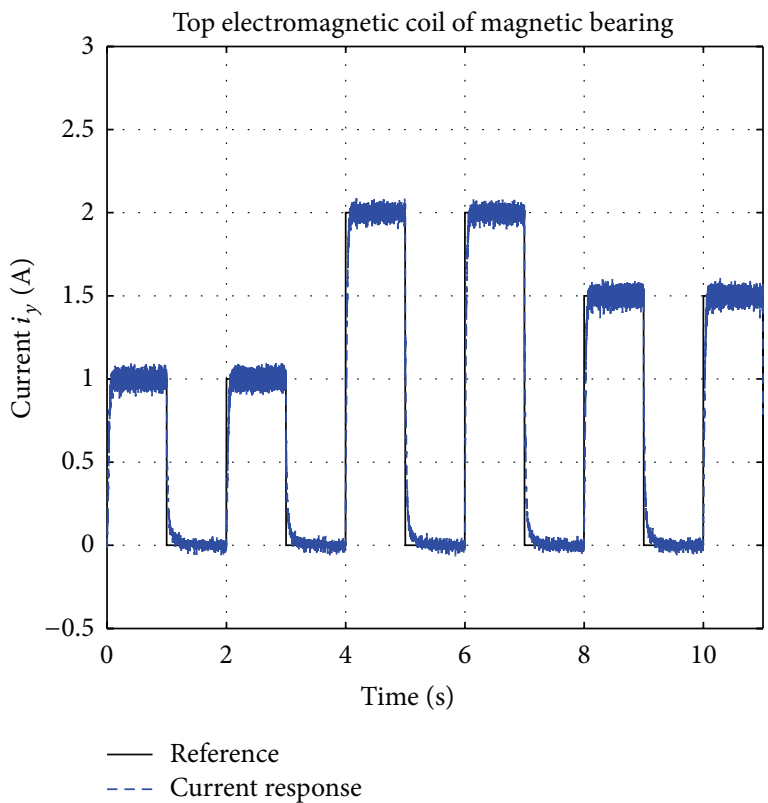

(c)

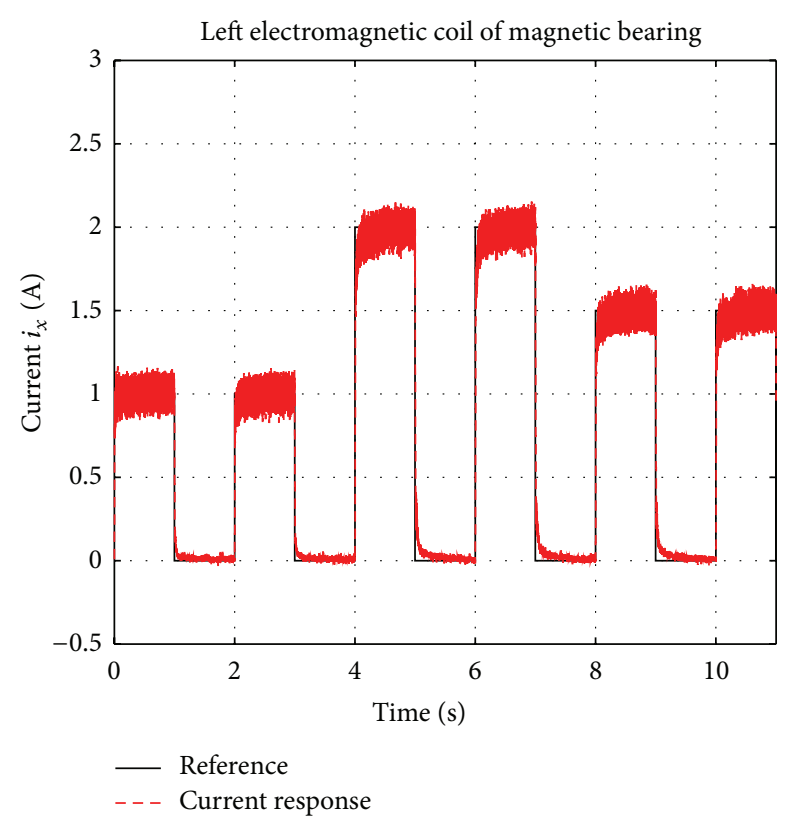

(b)

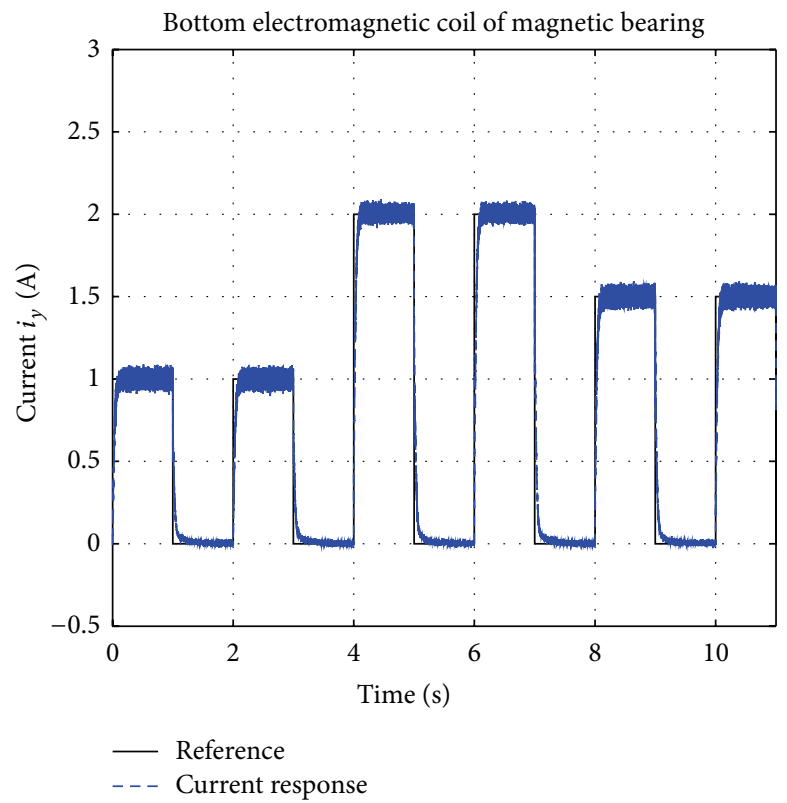

(d)

FIgURE 18: Current loop control of magnetic bearing with $x$-and $y$-axes.

The sampling rate of the 16-bit converter was $100 \mathrm{kHz}$ and the control cycle was approximately $0.1 \mathrm{~ms}$. A photograph of the experimental setup is presented in Figure 15. A current amplifier and current sensing circuit design for AMB system are indicated in Figure 16.

The AMB system and a fuzzy basis neural network were implemented using Matlab software; the parameters are listed in Table 1. The requisite interface was a PCI-1716 card comprising an A/D part with 16 channels and a digital input and output part with 16 channels. Matlab software was used to code the proposed controllers.
TABle 1: Parameters of an AMB system.

\begin{tabular}{lcc}
\hline 1 & Mass of shaft $(m)$ & $2.72 \mathrm{~kg}$ \\
2 & Nominal length of air gap $\left(x_{g}\right)$ & $0.5 \mathrm{~mm}$ \\
3 & Transverse moment of inertia of rotor $(J)$ & $0.013 \mathrm{kgm}^{2}$ \\
4 & Polar moment of inertia of rotor $\left(J_{z}\right)$ & $0.008 \mathrm{kgm}^{2}$ \\
5 & Displacement stiffness $\left(K_{x}\right)$ & $342478 \mathrm{~N} / \mathrm{m}$ \\
6 & Current stiffness $\left(K_{i}\right)$ & $171 \mathrm{~N} / \mathrm{A}$ \\
7 & Bias currents to be used $\left(i_{b}\right)$ & $1 \mathrm{~A}$ \\
\hline
\end{tabular}




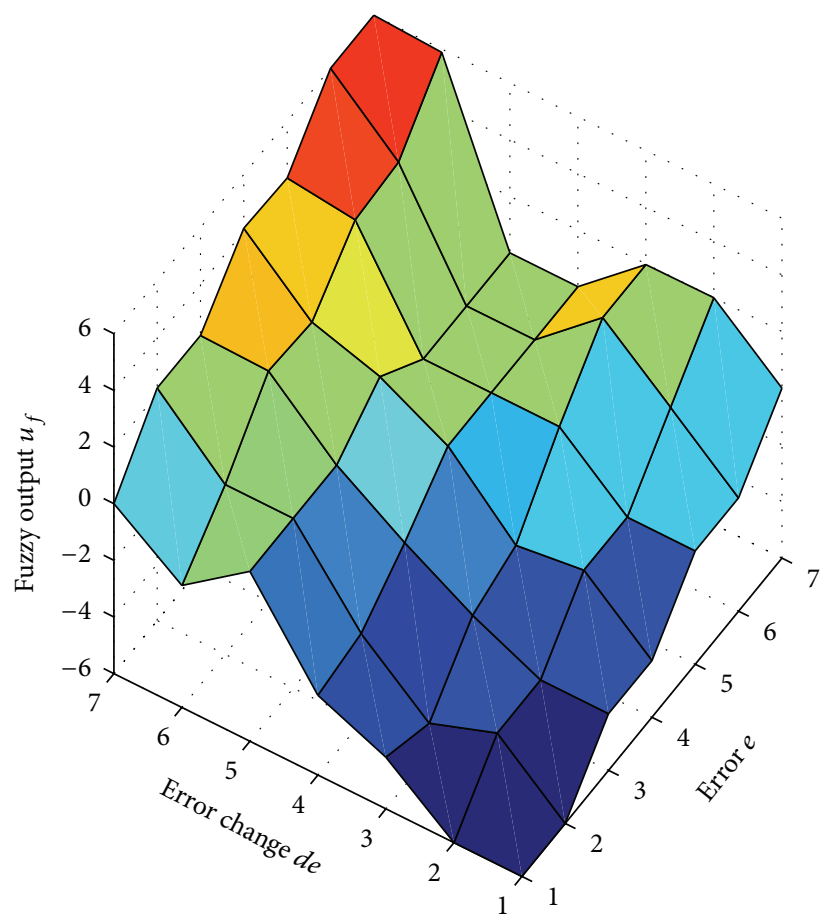

FIGURE 19: The control surface after adjusted NFC.

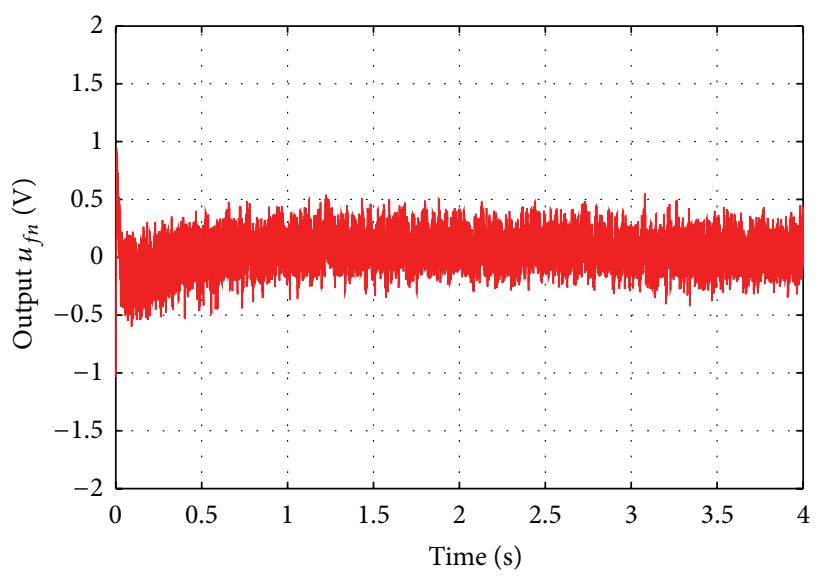

FIGURE 20: The output of RBFNN.

An experiment for the AMB system has been verified by the current-control loop, using FPID control and current amplifier (Figure 16). The model established for real time window target (RTWT) test for current-control loop using FPID controller is shown in Figure 17 (single electromagnetic coil), and the sample time is $0.1 \mathrm{~ms}$. The current responses of four electromagnetic in current-control loop at the same time are presented in Figure 18. From the results in Figure 18 we can see that the current response (four electromagnetic) is very close to the reference signal with the setting time about $0.01 \mathrm{~s}$. 2.

The system and controller parameters are listed in Table
The initial parameters in $w_{j}, b_{j}$, and $c_{j}$ were set as follows: $w_{j}=\left[\begin{array}{lll}0.5 & 0.5 & 0.5\end{array}\right] ; b_{j}=\left[\begin{array}{lll}40 & 0.5 & 0.5\end{array}\right]$; and $c_{j}=$

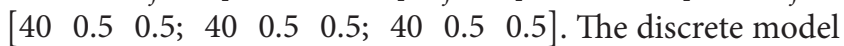
of the reference model was obtained as follows by using bilinear transformation at the sampling frequency of $1 \mathrm{kHz}$.

The AMB system included two pairs of electromagnets on the $x$ - and $y$-axes. The two pairs of electromagnets were simultaneously controlled using two NFCs. The experimental results are presented in Figures 19 and 20 and in Table 3. Figure 20 indicates that the practical output of the NFC converged from -1 to 1 .

Figure 21 indicates the rotor displacement of $x$ - and $y$ axes in the AMB system. The rotor displacement is small 


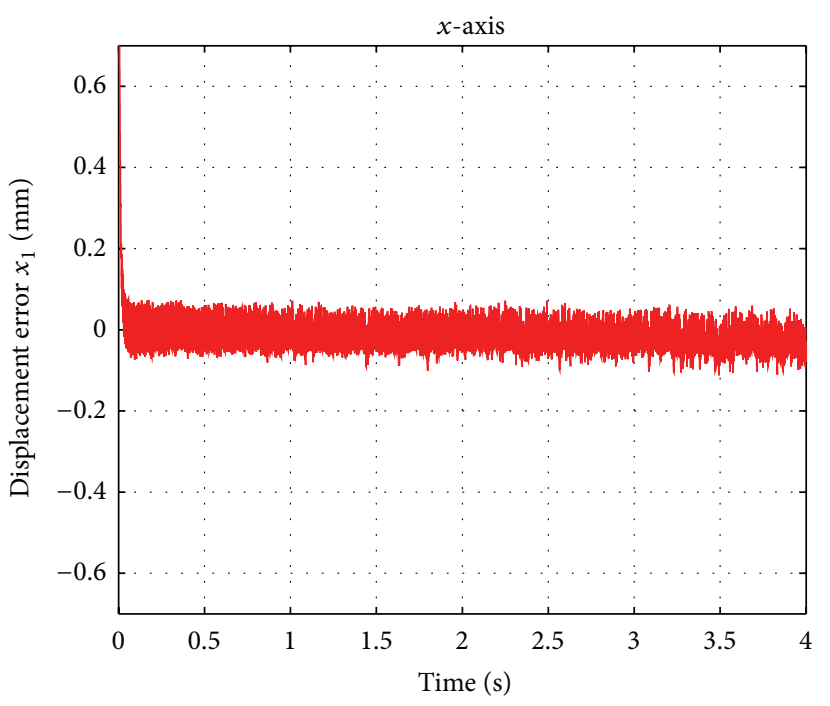

(a)

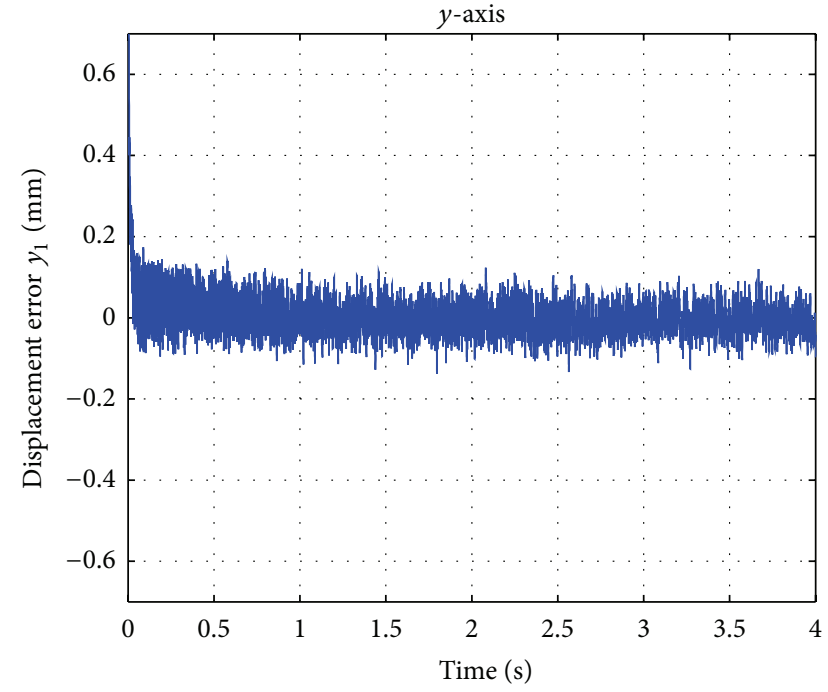

(b)

FIgURE 21: Rotor displacement of an AMB system with $x$ - and $y$-axes.

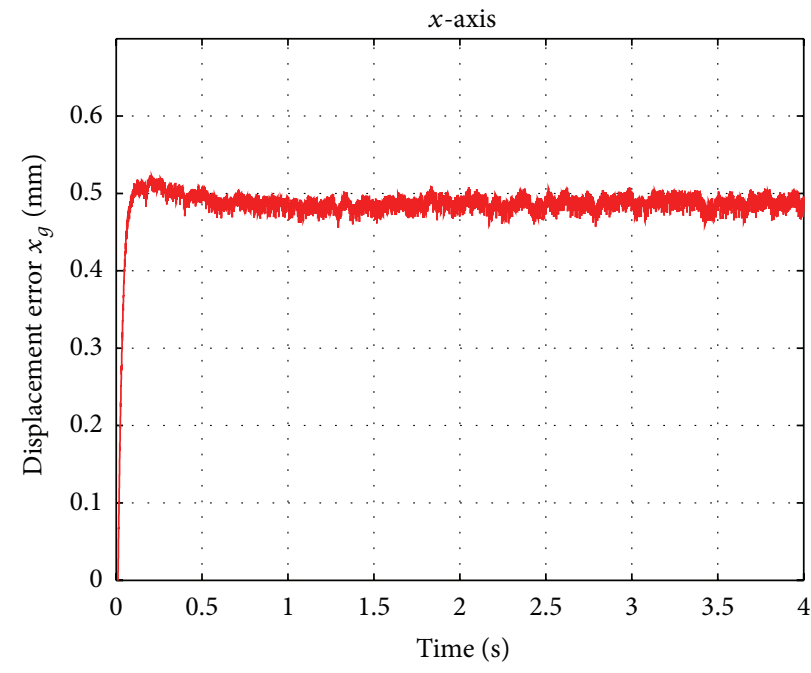

(a)

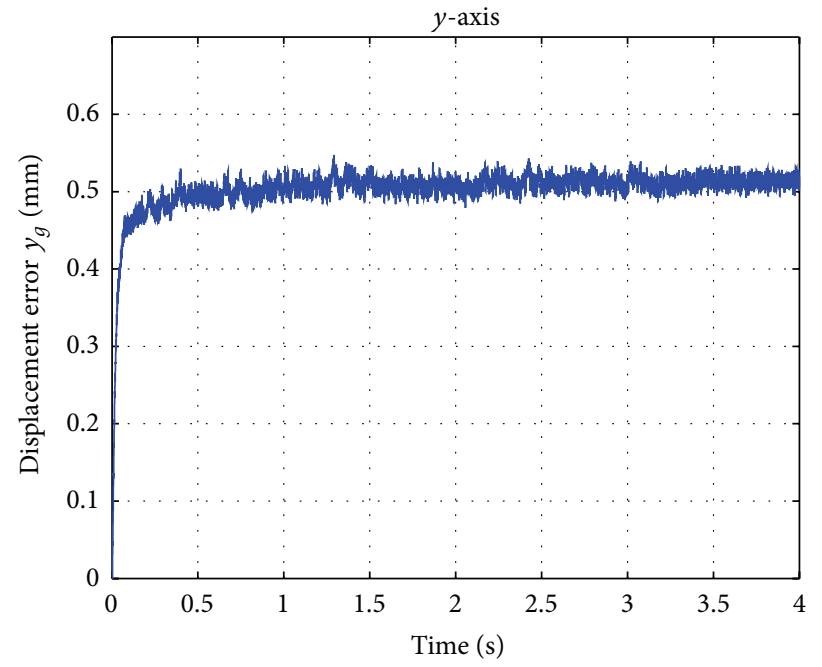

(b)

FIGURE 22: Step response of rotor position of AMB system.

about $0.12 \mathrm{~mm}$. In general, the rotor displacement in the horizontal direction is smaller than the rotor displacement in the vertical direction because of the effects of gravity on the $y$-axis. The response of rotor position of $x$ - and $y$-axes in AMB system covers the entire feasible region (Figure 22). Figures 23(a) and 23(b) show that the orbit of the rotor center is using an NFC at rotating speeds from $10000 \mathrm{rpm}$ to $15000 \mathrm{rpm}$. From rotating speed of $10000 \mathrm{rpm}$, the rotor displacement is small about 0.1 to $0.15 \mathrm{~mm}$ (Figure 23(a)). When the rotor rotates at a high speed $(15000 \mathrm{rpm})$, the rotor displacement increases about 0.19 to $0.22 \mathrm{~mm}$ (Figure 23(b)), but it is still in the permitted limits of nominal length of air gap $\left(x_{g}=0.5 \mathrm{~mm}\right)$. To evaluate the performance and characteristics of the RBFNN, we look at an AMB system controlled by an NFC. As we can see, it is unstable system before the first $1.2 \mathrm{~s}$. The parameters of a FLC are adjusted using a RBFNN. The whole controller is applied to the unbalanced vibration in an AMB system into effect after $1.2 \mathrm{~s}$. The results further demonstrate that a short rise time implies a short settling time, low overshoot, and a small steady-state error with external disturbance.

\section{Conclusions}

In this study, an NFC was developed to levitate rotor displacement in a highly unstable AMB system. The proposed 


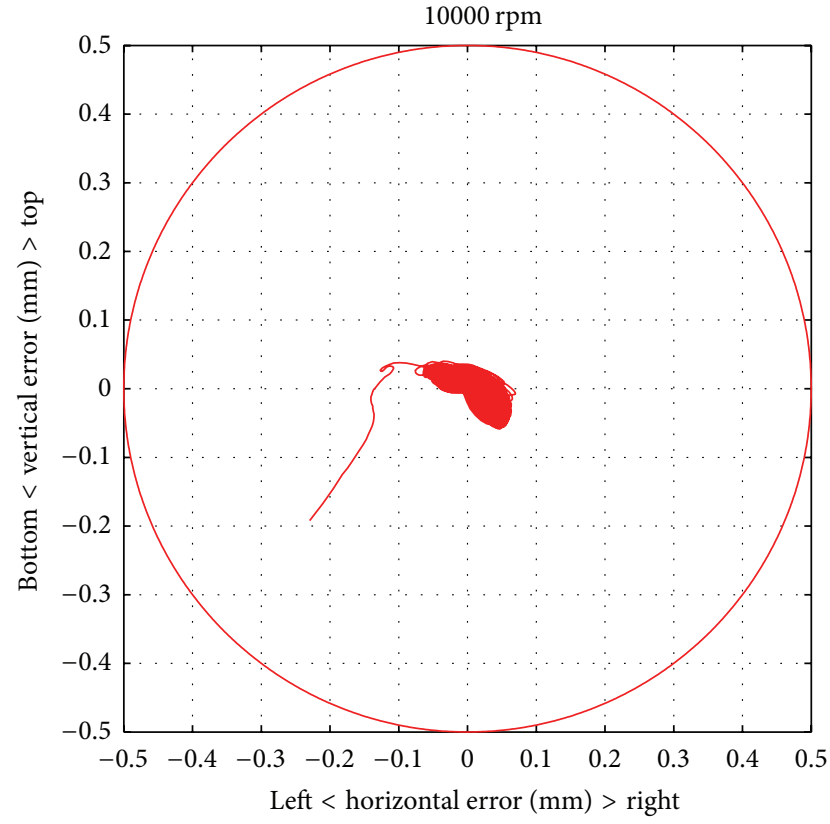

(a)

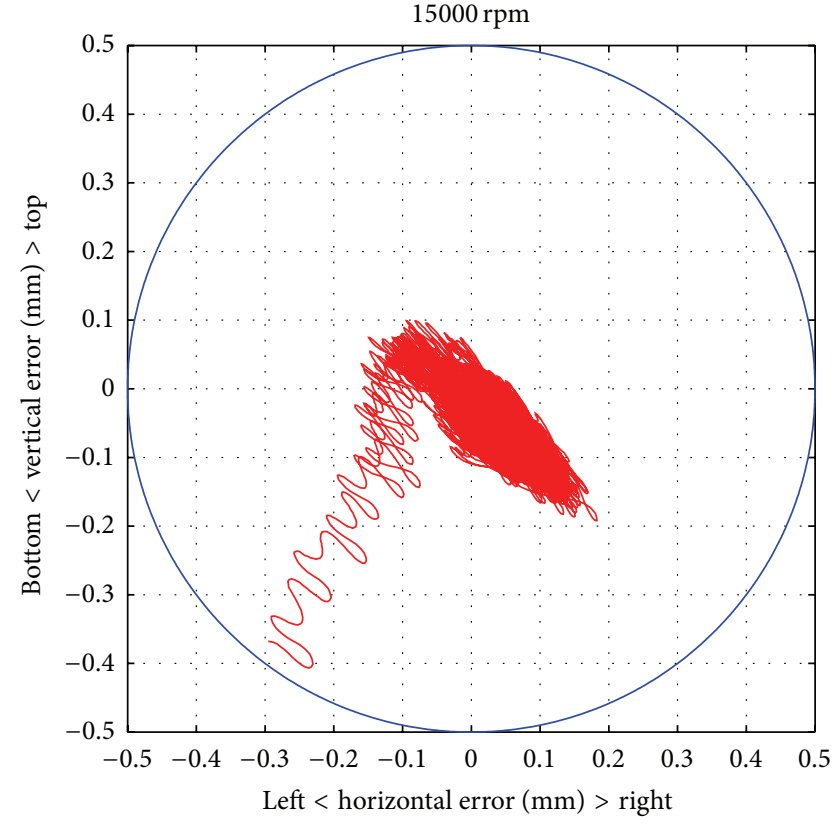

(b)

FIGURE 23: Orbits of rotor center $x$ - and $y$-axes.

TABLE 2: The controller parameters.

\begin{tabular}{cc}
\hline Parameters & Values \\
\hline Reference model & \\
$a_{0}$ & $98.10^{-6}$ \\
$a_{1}$ & $196.10^{-6}$ \\
$a_{2}$ & $98.10^{-6}$ \\
$b_{1}$ & -1.96 \\
$b_{2}$ & 0.96 \\
$\zeta$ & 1 \\
$\omega_{n}$ & $20 \mathrm{rad} / \mathrm{s}$ \\
PI controller & \\
$K_{p w}$ & 5.6 \\
$K_{i w}$ & 0.012 \\
NFC & \\
$\alpha$ & 0.07 \\
$\eta$ & 0.5 \\
\hline
\end{tabular}

method can also be used to improve the control performance of other nonlinear systems. The results indicated that the NFC responds well to a reference signal. The ABM system substantially reduced overshoot, shortened the adjustment time, accelerated the response, produced a more robust system, and improved its dynamic and static performance. The proposed controller can be feasibly applied to AMB systems with various external disturbances, and the effectiveness of the NFC with self-learning and self-improving capacities is
TABLE 3: Rule table after adjustment of neuron fuzzy controller.

\begin{tabular}{cccccccc}
\hline de & \multicolumn{7}{c}{$e$} \\
& $A_{0}$ & $A_{1}$ & $A_{2}$ & $A_{3}$ & $A_{4}$ & $A_{5}$ & $A_{6}$ \\
\hline$B_{0}$ & -6 & -6 & -4 & -4 & -2 & -2 & 0 \\
$B_{1}$ & -6 & -4 & -4 & -2 & -2 & 0 & 2 \\
$B_{2}$ & -4 & -4 & -2.084 & -2.109 & 0 & 2 & 2 \\
$B_{3}$ & -4 & -2 & -2.012 & 0 & 1.964 & 2 & 4 \\
$B_{4}$ & -2 & -2 & 0 & 2.113 & 2.065 & 4 & 4 \\
$B_{5}$ & -2 & 0 & 2.074 & 2.058 & 4.022 & 4 & 6 \\
$B_{6}$ & 0 & 2 & 2.055 & 4.027 & 4.024 & 6 & 6 \\
\hline
\end{tabular}

proven with RBFNN learning algorithm. This controller has been verified by the position control loop on a prototype AMB system.

\section{Conflict of Interests}

The authors declare that there is no conflict of interests regarding the publication of this paper.

\section{Acknowledgment}

The authors would like to thank the National Science Council and Bureau of Energy, Ministry of Economic Affairs, for financial support under Contract NSC 102-2221-E-212-007 and no. 102-D0624. 


\section{References}

[1] D. Johnson, G. V. Brown, and D. J. Inman, "Adaptive variable bias magnetic bearing control," in Proceedings of the American Control Conference (ACC '98), vol. 4, pp. 2217-2223, June 1998.

[2] H. W. Cho, C. H. Kim, J. M. Lee, and H. S. Han, "Design and characteristic analysis of small scale magnetic levitation and propulsion system for maglev train application," in Proceedings of the International Conference on Electrical Machines and Systems (ICEMS '11), pp. 1-5, August 2011.

[3] J. Kaloust, C. Ham, J. Siehling, E. Jongekryg, and Q. Han, "Nonlinear robust control design for levitation and propulsion of a Maglev system," IEE Proceedings-Control Theory and Applications, vol. 151, no. 4, pp. 460-464, 2004.

[4] J. Fei and S. Hou, "Robust adaptive fuzzy control for three-phase active power filter," in Proceedings of the IEEE 13th Workshop on Control and Modeling for Power Electronics (COMPEL '12), pp. 1-6, IEEE, Kyoto, Japan, July 2012.

[5] H.-C. Chen, "Optimal fuzzy pid controller design of an active magnetic bearing system based on adaptive genetic algorithms," in Proceedings of the International Conference on Machine Learning and Cybernetics (ICMLC '08), vol. 4, pp. 2054-2060, Kunming, China, July 2008.

[6] F. Matsumura and T. Yoshimoto, "System modeling and control design of ahorizontal-shaft magnetic-bearing system," IEEE Transactions on Magnetics, vol. 22, no. 3, pp. 196-203, 1986.

[7] S.-Y. Chen and F.-J. Lin, "Robust nonsingular terminal slidingmode control for nonlinear magnetic bearing system," IEEE Transactions on Control Systems Technology, vol. 19, no. 3, pp. 636-643, 2011.

[8] T. Dimond, P. Allaire, S. Mushi, Z. Lin, and S. Y. Yoon, "Modal tilt/translate control and stability of a rigid rotor with gyroscopics on active magnetic bearings," International Journal of Rotating Machinery, vol. 2012, Article ID 567670, 10 pages, 2012.

[9] S.-C. Chen and P.-C. Tung, "Application of a rule self-regulating fuzzy controller for robotic deburring on unknown contours," Fuzzy Sets and Systems, vol. 110, no. 3, pp. 341-350, 2000.

[10] B. Shi, L. Y. Wang, L. Peng, M. Xin, and Y. X. Huu, "A modified particle swarm optimization and radial basis function neural network hybrid algorithm model and its application," Intelligent Systems, vol. 1, pp. 134-138, 2009.

[11] N. Al-Holou, T. Lahdhiri, D. S. Joo, J. Weaver, and F. Al-Abbas, "Sliding mode neural network inference fuzzy logic control for active suspension systems," IEEE Transactions on Fuzzy Systems, vol. 10, no. 2, pp. 234-246, 2002.

[12] W. Wu, S. Zhong, and G. Zhou, "A study on PID intelligent optimization based on radial basis function neural networks," in Proceedings of the 3rd International Conference on Consumer Electronics, Communications and Networks (CECNet '13), pp. 57-60, Xianning, China, November 2013.

[13] S. C. Chen, N. V. Sum, and G. Chang, "Application of selftuning fuzzy PID controller on magnetic levitation system," in Proceedings of the 11th Taiwan Power Electronics Conference \& Exhibition, National Tsing Hua University, September 2012.

[14] P. S. Bhowmik, S. Pradhan, M. Prakash, and S. Roy, "Investigation of wavelets and radial basis function neural network for incipient fault diagnosis in induction motors," in Proceedings of the International conference on Circuits, Controls and Communications (CCUBE '13), pp. 1-5, Bengaluru, India, 2013.

[15] F. Guely and P. Siarry, "Gradient descent method for optimizing various fuzzy rule bases," in Proceedings of the 2nd IEEE
International Conference on Fuzzy Systems, vol. 2, pp. 1241-1246, San Francisco, Calif, USA, April 1993.

[16] G. Schweitzer, H. Bleuler, and A. Traxler, Active Magnetic Bearings, vdf Hochschulverlag AG, Zurich, Switzerland, 1994.

[17] K. Zdenko and B. Stiepan, Fuzzy Controller Design: Theory and Applications, CRC Press, 2005.

[18] S.-C. Chen, V.-S. Nguyen, D.-K. Le, and M.-M. Hsu, "ANFIS controller for an active magnetic bearing system," in Proceedings of the IEEE International Conference on Fuzzy Systems (FUZZ '13), pp. 1-8, Hyderabad, India, July 2013.

[19] F. Matsumura and T. Yoshimoto, "System modeling and control design of a horizontal shaft magnetic bearing system," IEEE Transactions on Magnetics, vol. 22, no. 3, pp. 196-203, 1986.

[20] M. Fumio, K. Hidehiko, and A. Yuji, "Fundamental equation for horizontal shaft magnetic bearing and its control system design," Fuzzy Sets and Systems, vol. 101, pp. 123-130, 2007.

[21] K. Nonami, "Vibration and control of flexible rotor supported by magnetic bearings," Magnetic Bearing, pp. 177-186, 1989.

[22] H. Baruh, Analytical Dynamics, McGraw-Hill International, 1999.

[23] S. Y. Yoon, Z. Lin, and P. E. Allaire, Control of Surge in Centrifugal Compressors by Active Magnetic Bearings: Theory and Implementation, Advances in Industrial Control, Springer, 2013.

[24] J. L. Meza, V. Santibáñez, R. Soto, and M. A. Llama, "Fuzzy selftuning PID semiglobal regulator for robot manipulators," IEEE Transactions on Industrial Electronics, vol. 59, no. 6, pp. 27092717, 2012.

[25] M. Maeda and S. Murakami, "A self-tuning fuzzy controller," Fuzzy Sets and Systems, vol. 51, no. 1, pp. 29-40, 1992.

[26] S. C. Chen, L. Y. Jyh, N. V. Sum, and S. M. Mao, "A novel fuzzy neural network controller for maglev system with controlledPM electromagnets," Intelligent Technologies and Engineering Systems, vol. 234, pp. 551-561, 2013.

[27] J. Y. Chen and H. L. Ying, "A self-tuning fuzzy controller design," in Proceedings of the IEEE International Conference on Neural Networks, vol. 3, pp. 1358-1362, 1995.

[28] Z. Q. Wu, P. Z. Wang, H. H. Teh, and S. S. Song, "A rule selfregulating fuzzy controller," Fuzzy Sets and Systems, vol. 47, no. 1, pp. 13-21, 1992.

[29] S.-Z. He, S.-H. Tan, F.-L. Xu, and P.-Z. Wang, "Fuzzy self-tuning of PID controllers," Fuzzy Sets and Systems, vol. 56, no. 1, pp. 3746, 1993.

[30] S.-Z. Gao, J. Yang, and J.-S. Wang, "D-FNN based modeling and BP neural network decoupling control of PVC stripping process," Mathematical Problems in Engineering, vol. 2014, Article ID 681259, 13 pages, 2014.

[31] F. A. Ruslan, A. M. Samad, Z. M. Zain, and R. Adnan, "Modelling flood prediction using Radial Basis Function Neural Network (RBFNN) and inverse model: a comparative study," in Proceedings of the IEEE International Conference on Control System, Computing and Engineering (ICCSCE '13), pp. 577-581, Mindeb, Mich, USA, December 2013.

[32] S. S. A. Ali, M. Moinuddin, K. Raza, and S. H. Adil, "An adaptive learning rate for RBFNN using time-domain feedback analysis," The Scientific World Journal, vol. 2014, Article ID 850189, 10 pages, 2014.

[33] M. Ali, S. Khan, M. Waleed, and Islamuddin, "Application of an intelligent self-tuning fuzzy PID controller on DC-DC buck converter," International Journal of Advanced Science and Technology, vol. 48, pp. 139-148, 2012. 
[34] M. Negnevitsky, Artificial Intelligence-A Guide to Intelligent Systems, 2nd edition, 2005.

[35] R.-E. Precup, M. L. Tomescu, and Ş. Preitl, "Fuzzy logic control system stability analysis based on Lyapunov's direct method," International Journal of Computers, Communications and Control, vol. 4, no. 4, pp. 415-426, 2009.

[36] M. A. Shoorehdeli, M. Teshnehlab, A. K. Sedigh, and M. A. Khanesar, "Identification using ANFIS with intelligent hybrid stable learning algorithm approaches and stability analysis of training methods," Applied Soft Computing, vol. 9, no. 2, pp. 833850, 2009.

[37] M. L. Tomescu and G. Petrov, "A stability analysis method for nonlinear systems with fuzzy logic controller," in Proceedings of the 8th International Symposium on Symbolic and Numeric Algorithms for Scientific Computing (SYNASC '06), pp. 141-150, IEEE, Timișoara, Romania, September 2006. 


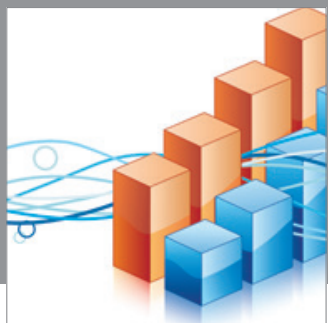

Advances in

Operations Research

mansans

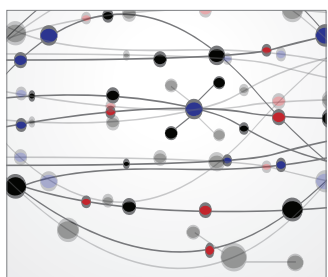

The Scientific World Journal
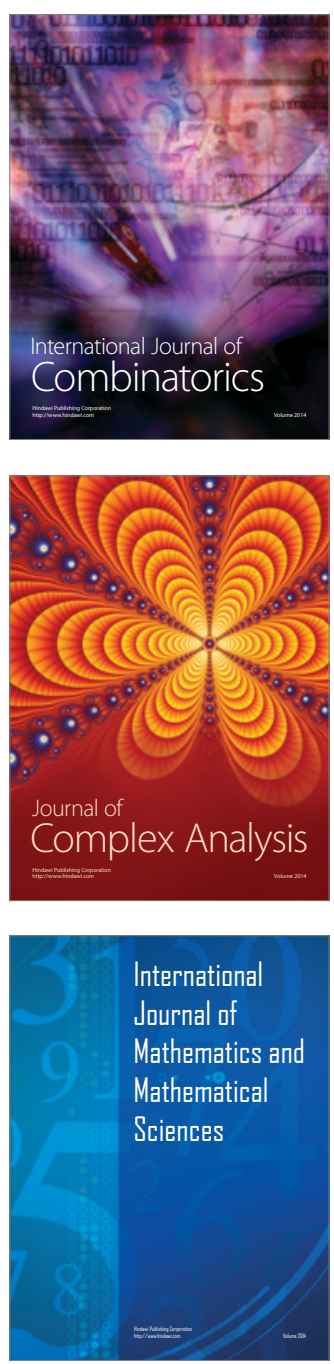
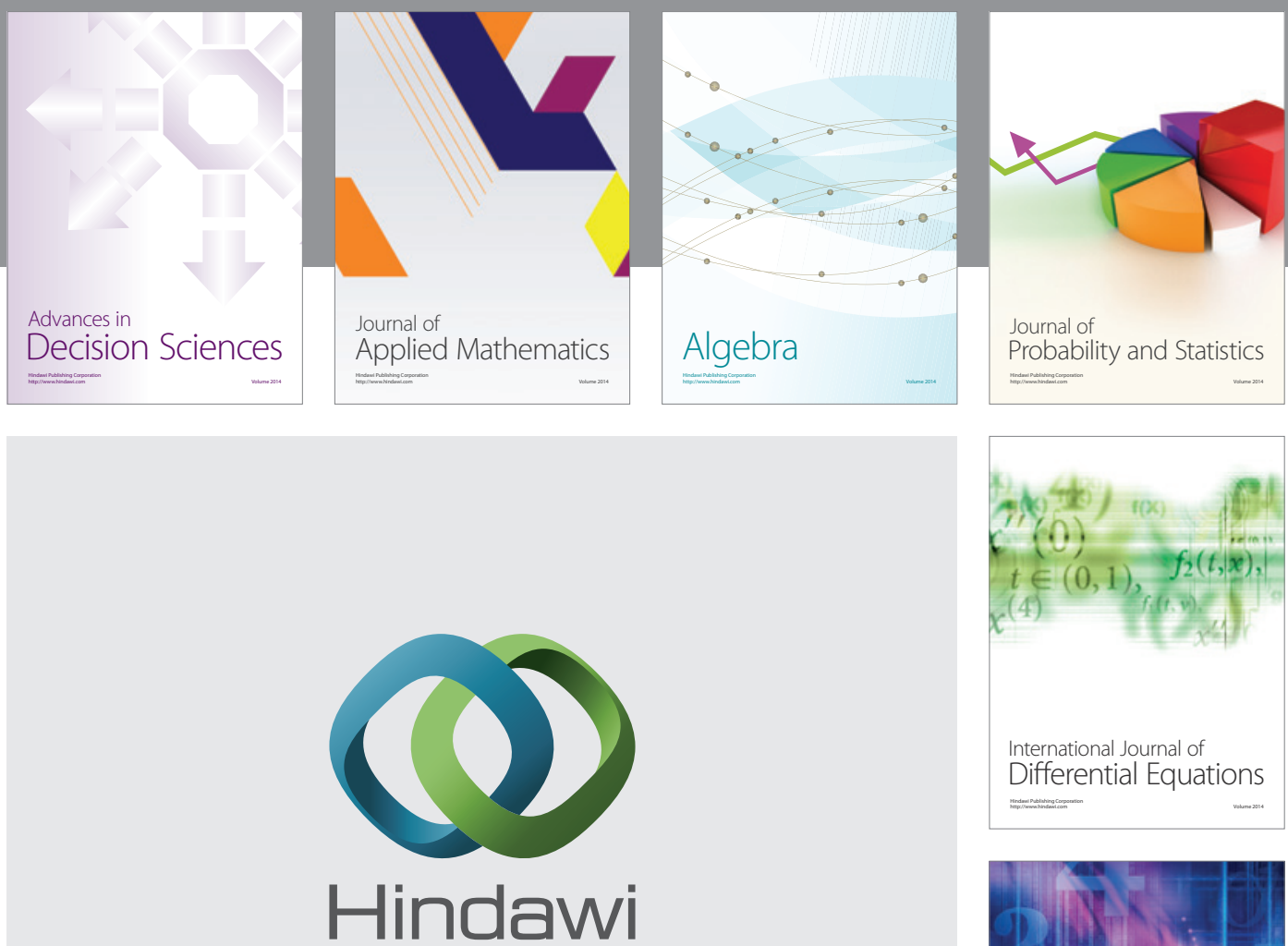

Submit your manuscripts at http://www.hindawi.com
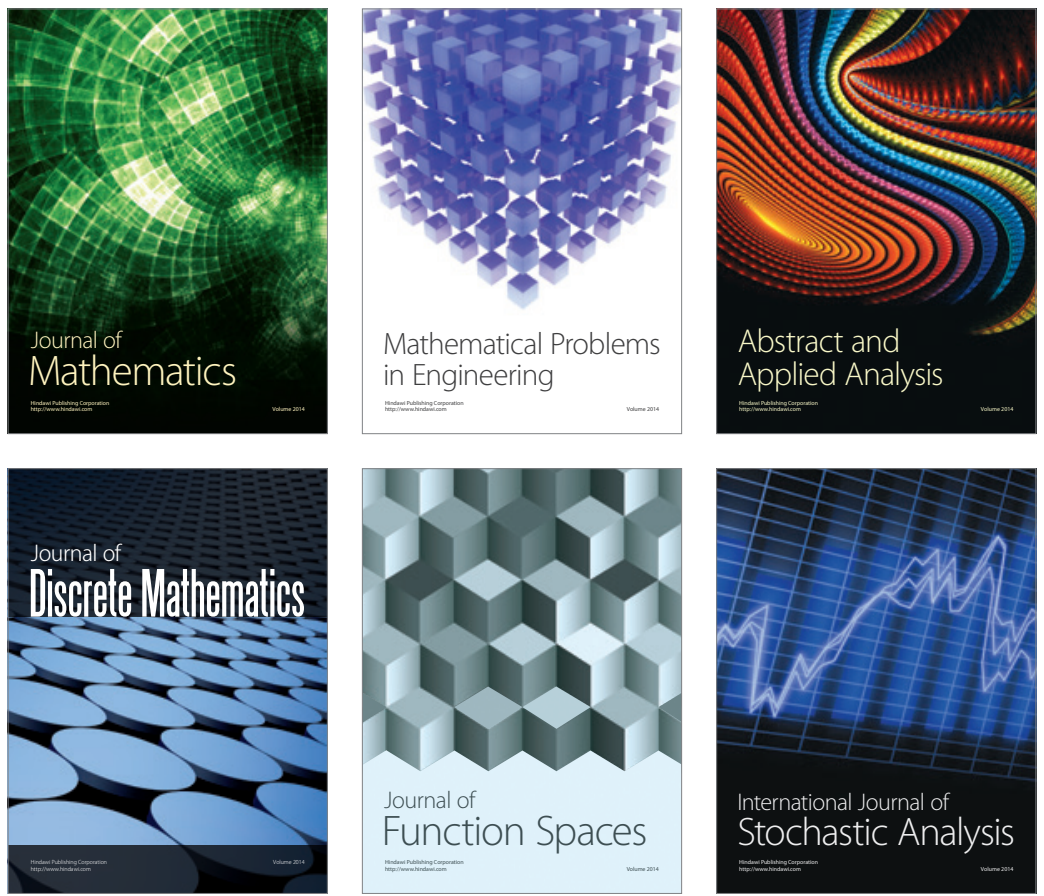

Journal of

Function Spaces

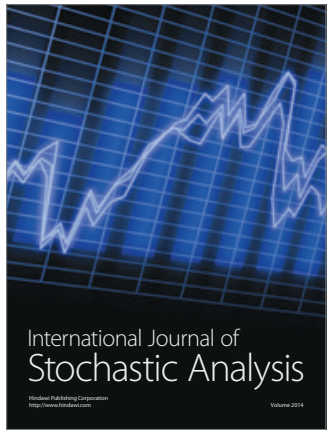

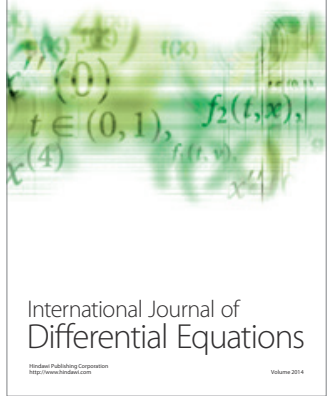
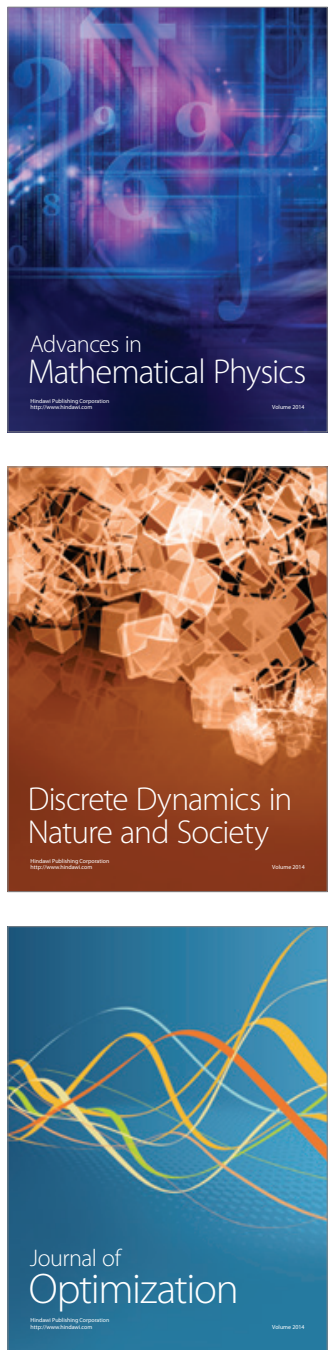